\title{
Recent Advances in Systemic Therapy for Hepatocellular Carcinoma in an Aging Society: 2020 Update
}

\author{
Masatoshi Kudo \\ Department of Gastroenterology and Hepatology, Kindai University Faculty of Medicine, \\ Osaka-Sayama, Japan
}

\section{Keywords}

Hepatocellular carcinoma - Molecular targeted drugs · Immune checkpoint inhibitors .

Combination immunotherapy $\cdot$ Elderly patients

\begin{abstract}
Systemic therapy for hepatocellular carcinoma (HCC) has changed markedly since the introduction of the molecular targeted agent sorafenib in 2007. Sorafenib increased the available treatment options for patients with extrahepatic spread and vascular invasion and improved survival in patients with advanced HCC; however, various shortcomings such as low response rates and relatively high toxicity (e.g., hand-foot skin reaction) prompted concerted efforts aimed at developing new molecular targeted agents to provide more treatment options and second-line agents for patients with disease progression or intolerance to sorafenib. Despite many attempts to develop new drugs between 2007 and 2016, all first-line and second-line clinical trials conducted during this period failed. However, between 2017 and 2019, 4 drugs (lenvatinib as a first-line agent and regorafenib, cabozantinib, and ramucirumab as secondline agents) emerged in quick succession from clinical trials and became available for clinical use. In addition, nivolumab and pembrolizumab were approved as second-line agents after sorafenib. A recent phase III trial (IMbrave150) showed that combination immunotherapy with atezolizumab plus bevacizumab increases overall survival compared with sorafenib therapy; Food and Drug Agency already approved this combination therapy, and worldwide approval is expected soon. This review describes the recent advances in systemic therapy and the use of tyrosine kinase inhibitors (sorafenib, lenvatinib, regorafenib, and cabozantinib), monoclonal antibodies (ramucirumab and bevacizumab), and immune checkpoint inhibitors (nivolumab, pembrolizumab, and atezolizumab) in elderly patients and the similarity of their efficacy and safety profiles to those in the general population.


Kudo: Systemic Therapy for HCC

\section{Introduction}

The established treatments for hepatocellular carcinoma (HCC) are resection, local ablation therapy, liver transplantation, transarterial chemoembolization (TACE), hepatic arterial infusion chemotherapy, and systemic therapies [1-3]. The Japan Society of Hepatology revised its clinical practice guidelines for HCC in 2017 [3]. This article reviews recent major advances and anticipated future developments in systemic therapy for HCC particularly in an aging society.

Systemic therapy for HCC has changed drastically since the introduction of the molecular targeted agent (MTA) sorafenib in 2007 [4, 5]. Although sorafenib improved the survival rates of patients with advanced HCC with vascular invasion and/or extrahepatic spread (EHS), shortcomings such as poor tumor-shrinking effects and relatively high toxicity (e.g., hand-foot skin reaction) prompted concerted efforts to develop new MTAs, immune checkpoint inhibitors (ICIs), or combinations of these to provide a greater number of options for patients. In addition, second-line agents were needed to treat progression in patients on sorafenib or in those intolerant to this agent. Several attempts to develop new drugs were made between 2007 and 2016; however, many clinical trials in the first-line and second-line settings conducted during this period failed [6-30] (Table 1). This changed starting on 2017 and through 2019, during which 4 drugs (lenvatinib as a first-line agent and regorafenib, ramucirumab, and cabozantinib as second-line agents) emerged in quick succession from clinical trials and became available for clinical use [16, 25, 28, 29] (Table 1). A recent phase III trial (IMbrave150) showed that combination immunotherapy with atezolizumab plus bevacizumab (Atezo + Beva) improves all the efficacy outcomes, including overall survival (OS), progression-free survival (PFS), objective response rate (ORR), disease control rate (DCR), and patient-reported outcome of quality of life compared with those obtained with sorafenib monotherapy [19].

The efficacy of TACE plus sorafenib combination therapy was first demonstrated in 2020 [31] (Table 2), whereas 5 previous TACE combination trials with sorafenib/brivanib/orantinib showed negative results [32-36]. In light of the recent global tendency in aging societies such as Japan, in which the number of elderly HCC patients is steadily increasing regarding both incidence and mortality [37, 38] (Fig. 1), it is important to understand the safety and efficacy of currently available systemic therapeutic agents. This article reviews recent advances in systemic therapy for HCC as well as the safety and efficacy of systemic therapy in elderly patients.

\section{Molecular Targeted Agents: First-Line Drugs}

\section{Sorafenib}

Sorafenib is an oral kinase inhibitor that exerts antitumor effects by suppressing tumor proliferation through inhibition of the serine/threonine kinases C-Raf, wild-type B-RAF, and mutant (V600E) B-RAF, which are all components of the Raf/MEK/ERK pathway (MAP kinase pathway) downstream of vascular endothelial growth factor receptor (VEGFR), plateletderived growth factor receptor (PDGFR), and epidermal growth factor receptor; the drug also suppresses angiogenesis by inhibiting tyrosine kinases such as VEGFR1, VEGFR2, VEGFR3, PDGFR $\beta$, RET, and fms-related tyrosine kinase-3. Two large phase III trials (the SHARP and Asia-Pacific trials) $[4,5]$ showed that sorafenib prolongs OS significantly over placebo, and the drug is currently the standard therapy for advanced HCC. However, because of its shortcomings (poor antitumor effects and relatively high toxicity), developing a new MTA with superior efficacy and/or lower toxicity has been a critical issue.

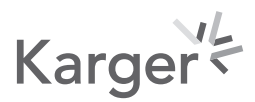




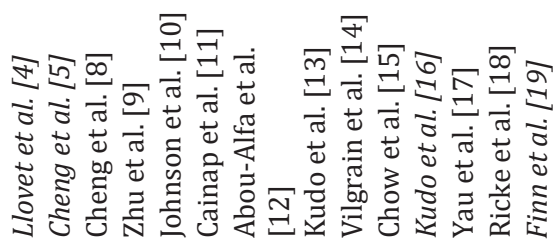

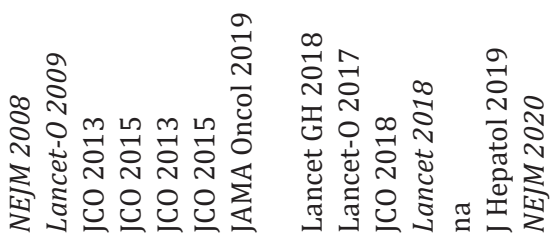

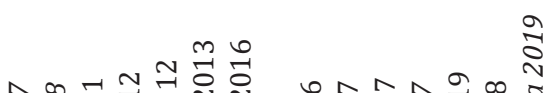

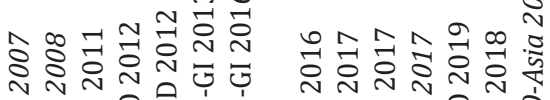
N

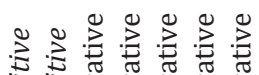
s.

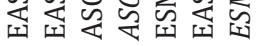

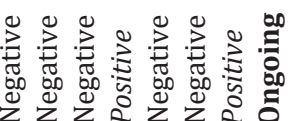

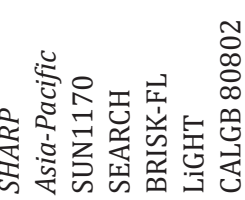
₹ ๙

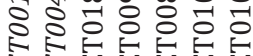
zzzzzz
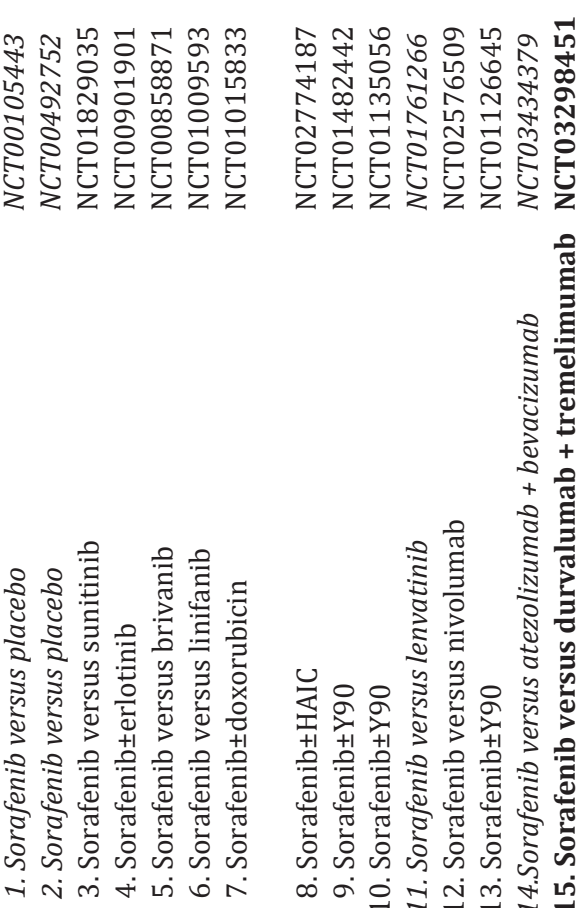

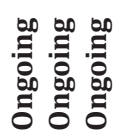

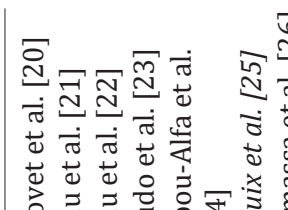

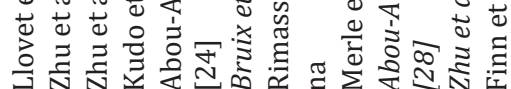

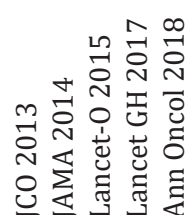

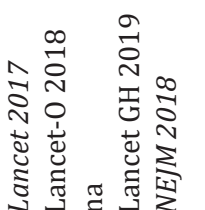

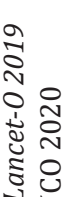
년

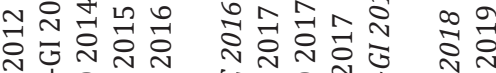

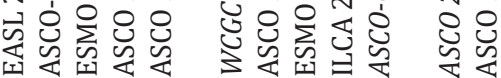

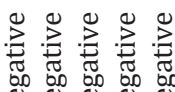

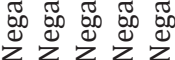

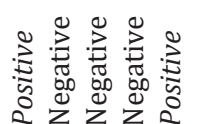

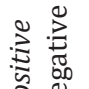
盆

\section{苞}

号古 窇矛焉

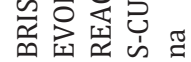

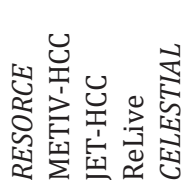

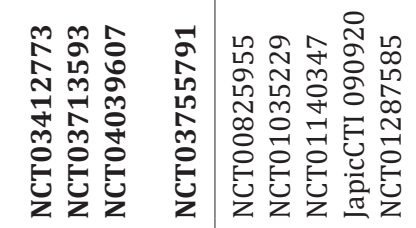

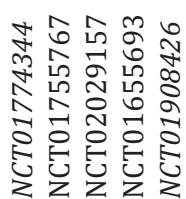

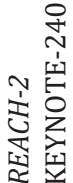

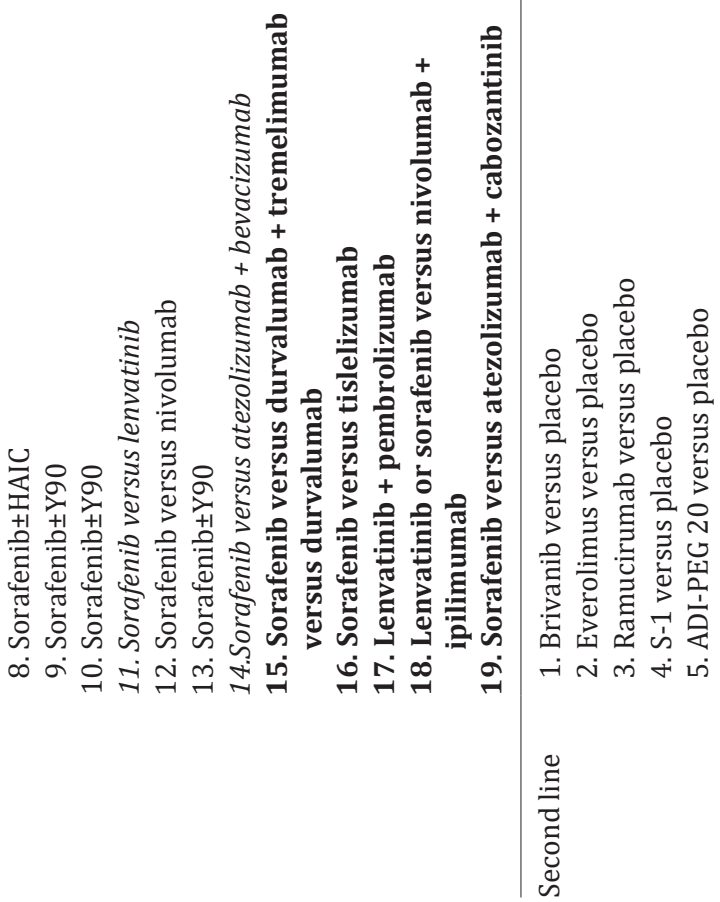

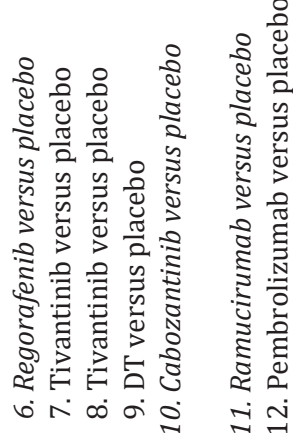




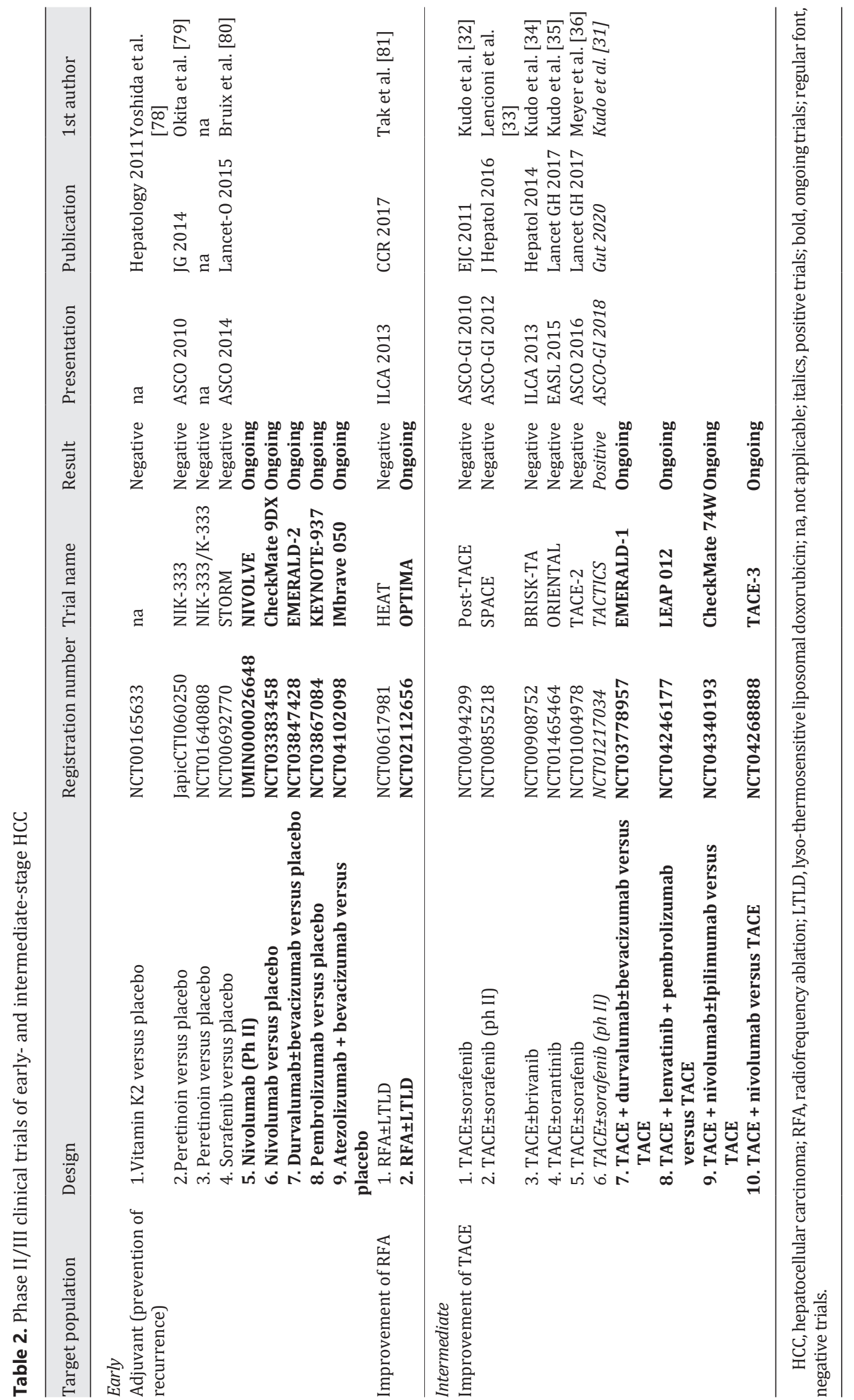


Fig. 1. a Incidence of HCC in Japan reported in 2015: incidence of HCC in male patients was most frequently observed in the range of 75-79 years and that in female patients was >85 years old. b Mortality of HCC in Japan reported in 2018: the most frequent death age is over 80 years both in male and female patients. HCC, hepatocellular carcinoma.

\begin{tabular}{l|l}
\hline DOI: 10.1159/000511001 & $\begin{array}{l}\text { (c) 2020 The Author(s). Published by S. Karger AG, Basel } \\
\text { www.karger.com/lic }\end{array}$ \\
\hline
\end{tabular}

Kudo: Systemic Therapy for HCC
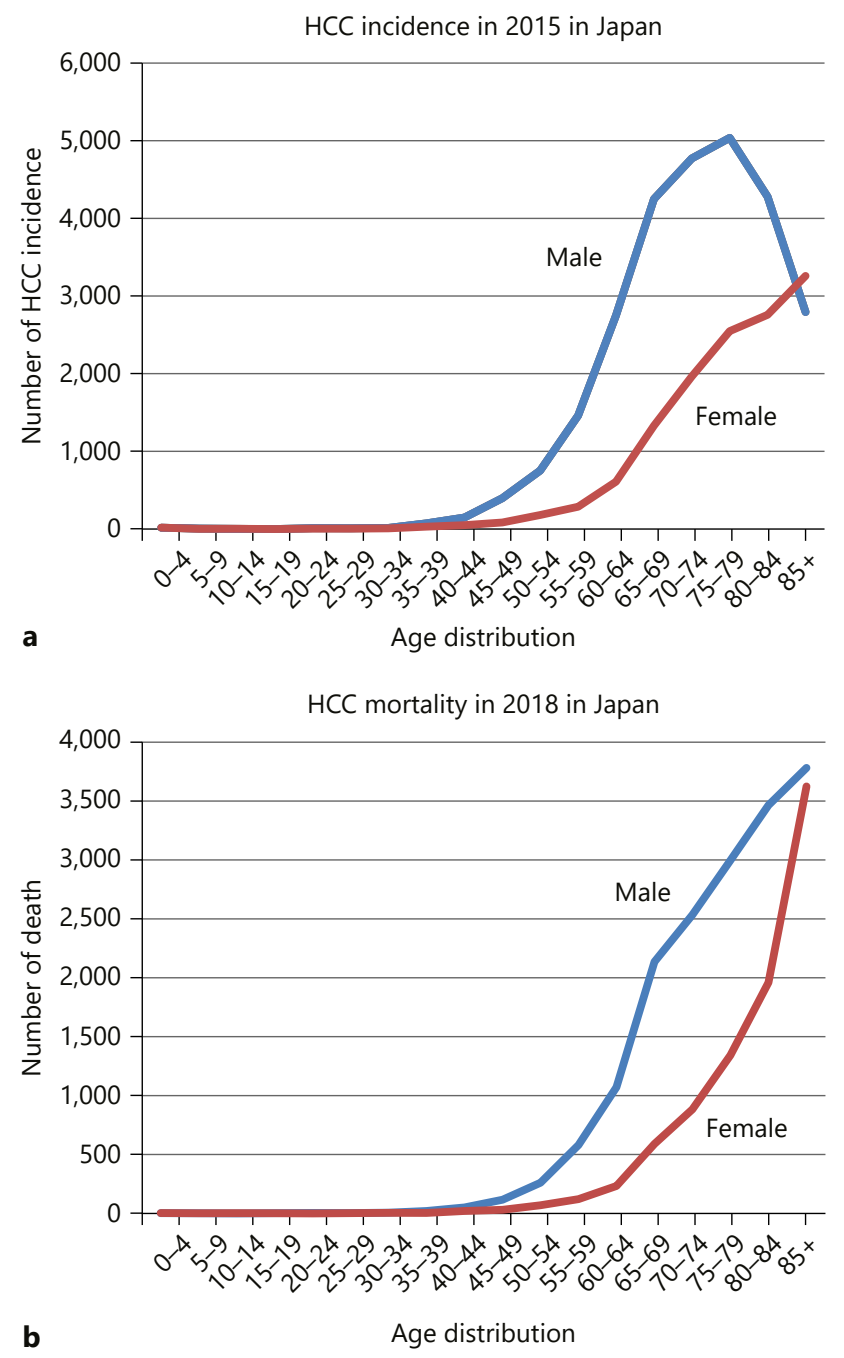

HCC mortality in 2018 in Japan

b

The safety and efficacy of sorafenib in elderly patients need to be investigated in detail. Although subgroup analysis by age was not performed in the SHARP trial [4], it was included in the Asia-Pacific trial [5]. According to the Asia-Pacific trial, OS in elderly patients ( $\geq 65$ years) was longer in the sorafenib group than in the placebo group (hazard ratio [HR] $=0.74$; $95 \%$ confidence interval [CI]: $0.32-1.70$ ). HR for OS in elderly patients ( $\geq 65$ years) was similar to that in nonelderly patients ( $\mathrm{HR}=0.66 ; 95 \% \mathrm{CI}: 0.47-0.92$ ), suggesting that the efficacy of sorafenib in elderly patients is similar to that in nonelderly patients.

In the real-world setting, Jo et al. [39] reported that there were no significant differences in OS, ORR, and frequency and severity of drug-related adverse events (AEs) between elderly patients ( $\geq 80$ years) and nonelderly patients ( $<80$ years) in both the entire study cohort $(n=$ $185)$ and the propensity-matched cohort $(n=48)$. Ziogas et al. [40] reported similar results, showing that the safety and efficacy of sorafenib did not differ between elderly patients $(\geq 75$ years) ( $n=39)$ and nonelderly patients ( $<75$ years) $(n=151)$. In a propensity-matched study, Nishikawa et al. [41] reported that OS in elderly patients ( $\geq 80$ years) $(n=132)$ was similar to that in nonelderly patients ( $<80$ years) $(n=132)$ (9.3 and 8.8 months, respectively; $p=$ 
0.8247). PFS in elderly patients was also similar to that in nonelderly patients (3.8 [95\% CI: 2.2-5.4] and 3.4 [95\% CI: 3.1-3.7] months, respectively, $p=0.668$ ). DCR and ORR were also similar in both age subgroups. Treatment-related grade 3 or higher serious AEs were observed in $28.5 \%$ of elderly patients and $24.7 \%$ of nonelderly patients ( $p=0.385)$. Therefore, the authors concluded that there were no significant differences in efficacy and safety between elderly and nonelderly patients.

However, Williet et al. [42] reported that tolerability to sorafenib is low in elderly patients ( $\geq 80$ years) and OS is poor in elderly patients ( $\geq 85$ years). Similarly, Morimoto et al. [43] reported higher discontinuation rates and lower OS in elderly patients ( $\geq 75$ years) $(n=24)$ than in nonelderly patients $(n=52)$ with a starting dose of sorafenib of $800 \mathrm{mg}$. However, safety and efficacy were comparable between the elderly and nonelderly populations treated with a half-dose regimen, which is consistent with the results reported by Montella et al. [44].

\section{Lenvatinib: Overview of the REFLECT Trial Results}

The REFLECT trial was the first positive phase III clinical trial in the first-line setting undertaken during a 10-year period in which 8 other trials were negative. Lenvatinib is an oral kinase inhibitor that selectively inhibits receptor tyrosine kinases involved in tumor angiogenesis and malignant transformation (e.g., VEGFR1, VEGFR2, VEGFR3, fibroblast growth factor receptor [FGFR]1, FGFR2, FGFR3, FGFR4, PDGFR $\alpha$, KIT, and RET). Because it is a particularly strong inhibitor of FGFR4, it is useful for treating high malignancy grade or poorly differentiated HCC. A single-arm phase II trial of lenvatinib in advanced HCC showed excellent results: the time to progression (TTP) was 7.4 months and OS was 18.7 months [45]. This was followed by the phase III REFLECT trial comparing sorafenib and lenvatinib [16].

The REFLECT trial was a global phase III trial assessing the noninferiority of lenvatinib to sorafenib. Patients were randomized to a lenvatinib or a sorafenib arm at a 1:1 ratio and stratified by ethnicity (Asian or non-Asian), vascular invasion and/or EHS (presence or absence), Eastern Cooperative Oncology Group performance status (ECOG PS; 0 or 1), and body weight $(<60$ or $\geq 60 \mathrm{~kg}$ ). Noninferiority of OS was set as the primary endpoint, and the noninferiority margin was set at 1.08. PFS, TTP, ORR, and safety were evaluated as secondary endpoints.

The proportion of patients with HCC caused by hepatitis $\mathrm{C}$ was balanced in favor of the sorafenib arm (27 vs. 19\% in the lenvatinib arm) [16]. Conversely, the proportion of patients in the lenvatinib arm with HCC caused by hepatitis B was $53 \%$ compared with $48 \%$ in the sorafenib arm. The proportion of patients with alpha-fetoprotein (AFP) levels $\geq 200 \mathrm{ng} / \mathrm{mL}$ was also balanced favorably toward the sorafenib arm (39 vs. $46 \%$ in the lenvatinib arm).

The primary endpoint for OS was 13.6 months in the lenvatinib arm and 12.3 months in the sorafenib arm. The upper limit of the $95 \%$ CI of the HR was $0.92(0.79-1.06)$ and was below the prespecified noninferiority margin of 1.08, which demonstrated statistically the noninferiority of lenvatinib with respect to OS [16]. PFS (7.4 months in the lenvatinib arm vs. 3.7 months in the sorafenib arm), TTP (8.9 vs. 3.7 months), and ORR ( 24.1 vs. 9.2\%) per investigator assessment using the modified Response Evaluation Criteria in Solid Tumors ( $m R E C I S T)$ were better in the lenvatinib arm than in the sorafenib arm (odds ratio [OR] = 3.13; 95\% CI, 2.15-4.56, $p<0.0001$ ), demonstrating the significantly better antitumor effect of lenvatinib (Table 3) [16]. Another important finding was that tumor shrinkage or necrotic effects were excellent in the lenvatinib arm, as illustrated by the ORR per independent imaging review using mRECIST ( $40.6 \%$ in the lenvatinib arm vs. $12.4 \%$ in the sorafenib arm) (OR = 5.01; 95\% CI: 3.59-7.01; $p<0.0001)[16,46]$. Since objective response per mRECIST predicts better survival, lenvatinib provided a survival benefit in more patients with advanced HCC [47]. The good antitumor effect suggested by the PFS, TTP, and ORR results was reproduced exactly in an independent imaging review using RECIST 1.1 [16].

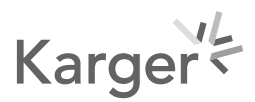


Table 3. Efficacy results of the phase 3 REFLECT trial (mRECIST)

\begin{tabular}{lccl}
\hline & Lenvatinib $(n=478)$ & Sorafenib $(n=476)$ & HR, $p$ value \\
\hline OS, M (95\% CI) & $13.6(12.1-14.9)$ & $12.3(10.4-13.9)$ & HR 0.92 (95\% CI: 0.79-1.06) \\
PFS, M (95\% CI) & $7.3(5.6-7.5)$ & $3.6(3.6-3.7)$ & HR 0.64 (95\% CI: 0.55-0.75) \\
& & & $p<0.0001$ \\
TTP, M (95\% CI) & $7.4(7.2-9.1)$ & $3.7(3.6-3.9)$ & HR $0.60(95 \%$ CI: 0.51-0.71) \\
& & & $p<0.0001$ \\
Objective response, $n(\%)^{*}$ & $10(2)$ & $4(1)$ & \\
CR & $184(38)$ & $55(12)$ & \\
PR & $159(33)$ & $219(46)$ & \\
SD & $79(17)$ & $152(32)$ & $p<0.0001$ \\
PD & $194(40.6)$ & $59(12.4)$ & $p<0.0001$ \\
ORR & $353(73.8)$ & $278(58.4)$ & \\
DCR & & &
\end{tabular}

OS, overall survival; PFS, progression-free survival; TTP, time to progression; CR, complete response; PR, partial response; SD, stable disease; PD, progressive disease; mRECIST, modified Response Evaluation Criteria in Solid Tumors. * Independent imaging review per mRECIST.

Because patients were not stratified by AFP, the lenvatinib arm included a higher proportion of patients with AFP $\geq 200 \mathrm{ng} / \mathrm{mL}$. After correcting this AFP imbalance by analysis of covariance during OS analysis, lenvatinib was statistically superior to sorafenib with respect to OS (HR $=0.856$; 95\% CI: 0.736-0.995; nominal $p$ value $=0.0342$ ) [16]. Thus, this trial could have demonstrated superiority if patients had been stratified by AFP [48].

Lenvatinib also yielded longer OS in patients with high baseline AFP ( $\geq 200 \mathrm{ng} / \mathrm{mL})$, which is a poor prognostic indicator, as illustrated by a HR of 0.78 (95\% CI: $0.63-0.98)$. The treatment duration in the lenvatinib arm was 5.7 months and that in the sorafenib arm was 3.7 months, indicating that patients were able to continue lenvatinib treatment longer than sorafenib. Regarding AEs, lenvatinib and sorafenib showed different safety profiles; lenvatinib was associated with a lower incidence of subjective AEs such as hand-foot skin reactions and diarrhea. However, the REFLECT trial excluded HCC patients with tumor occupancy $\geq 50 \%$, main portal vein invasion, and biliary tract invasion, who may not benefit from systemic therapy.

The above results statistically demonstrate the noninferiority of lenvatinib to sorafenib with respect to OS; in addition, the trial showed statistically and clinically significant improvements in all secondary endpoints (PFS, TTP, and ORR), which demonstrates the efficacy of lenvatinib as a first-line drug for unresectable HCC. In light of these results, HCC was added as another indication for lenvatinib worldwide in 2018.

Regarding the efficacy and safety of lenvatinib in elderly patients, specific subanalysis of age subgroups was not performed in the REFLECT trial. However, the OS HR in patients $\geq 65$ years in the REFLECT trial was 0.84 (95\% CI: 0.66-1.07), which is similar to that in patients $<65$ years ( $\mathrm{HR}=0.94$ [95\% CI: $0.77-1.15]$ ). The PFS HR in patients $\geq 65$ years in the lenvatinib arm was 0.61 (95\% CI: $0.48-0.78$ ), which is similar to that in patients $<65$ years ( $\mathrm{HR}=0.67$; 95\% CI: $0.55-0.82$ ). The TTP HR in patients $\geq 65$ years old in the lenvatinib arm over the sorafenib arm was 0.57 (95\% CI: 0.44-0.74), which is also similar to that in patients $<65$ years (HR $=0.63 ; 95 \% \mathrm{CI}: 0.52-0.78$ ) [16]. The OR for ORR in patients $\geq 65$ years was 2.96 (95\% CI: $1.66-5.29$ ) (25.5\% in lenvatinib and 9.3\% in sorafenib), which is similar to that in patients $<65$ years (OR $=2.97 ; 95 \% \mathrm{CI}: 1.78-4.94)$ (23.0\% in lenvatinib and $9.2 \%$ in sorafenib) [16].

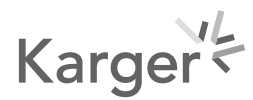


Kudo: Systemic Therapy for HCC

Table 4. Efficacy results of the phase 3 RESORCE trial (mRECIST)

\begin{tabular}{|c|c|c|c|}
\hline & Regorafenib $(n=379)$ & Placebo $(n=194)$ & HR, $p$ value \\
\hline OS, M (95\% CI) & $10.6(9.1-12.1)$ & $7.8(6.3-8.8)$ & $\begin{array}{l}\text { HR } 0.63 \text { (95\% CI: } 0.50-0.79) \\
p<0.0001\end{array}$ \\
\hline PFS, M (95\% CI) & $3.1(2.8-4.2)$ & $1.5(1.4-1.6)$ & $\begin{array}{l}\text { HR } 0.46 \text { (95\% CI: } 0.37-0.56) \\
p<0.0001\end{array}$ \\
\hline TTP, M (95\% CI) & $3.2(2.9-4.2)$ & $1.5(1.4-1.6)$ & $\begin{array}{l}\text { HR } 0.44 \text { (95\% CI: } 0.36-0.55) \\
p<0.0001\end{array}$ \\
\hline \multicolumn{4}{|c|}{ Objective response, $n(\%)^{*}$} \\
\hline $\mathrm{CR}$ & $2(1)$ & 0 & \\
\hline PR & $38(10)$ & $8(4)$ & \\
\hline SD & $206(54)$ & $62(32)$ & \\
\hline PD & $86(23)$ & $108(56)$ & \\
\hline ORR & $40(11)$ & $8(4)$ & $p=0.0047$ \\
\hline DCR & $247(65)$ & $70(36)$ & $p<0.0001$ \\
\hline
\end{tabular}

OS, overall survival; PFS, progression-free survival; TTP, time to progression; CR, complete response; PR, partial response; SD, stable disease; PD, progressive disease; mRECIST, modified Response Evaluation Criteria in Solid Tumors. * Independent imaging review per mRECIST.

In the real-world clinical setting, Tada et al. [49] compared the safety and efficacy of lenvatinib between elderly patients ( $\geq 75$ years) $(n=50)$ with unresectable HCC and nonelderly patients $(n=50)$ using propensity score matching. They concluded that there were no significant differences between elderly and nonelderly patients in the frequency of AEs. Similarly, regarding OS and PFS, there were no significant differences between elderly and nonelderly patients regarding OS (HR $=0.972$; 95\% CI: 0.374-2.529) and PFS (HR $=1.362 ; 95 \% \mathrm{CI}: 0.687-$ 2.700) [49]. Similarly, Tsuchiya et al. [50] reported that OS, PFS, treatment duration, ORR, and DCR were not significantly different between lenvatinib-treated elderly patients ( $\geq 75$ years) and nonelderly patients ( $<75$ years). These results suggest that the efficacy and safety of lenvatinib are similar in elderly patients ( $\geq 75$ years) and in nonelderly patients ( $<75$ years).

\section{Molecular Targeted Agents: Second-Line Drugs}

\section{Regorafenib: Overview of the RESORCE Trial}

Regorafenib is an oral inhibitor of multiple protein kinases such as VEGFR1, VEGFR2, VEGFR3, TIE2, PDGFR $\beta$, FGFR, KIT, RET, RAF-1, and B-RAF [51]. Its molecular structure is nearly identical to that of sorafenib except for the addition of a fluorine bond, resulting in a toxicity profile similar to that of sorafenib. It was investigated in a phase III placebo-controlled trial (the RESORCE trial) that exclusively enrolled patients who progressed on and were able to tolerate sorafenib at $\geq 400 \mathrm{mg} /$ day for at least 20 days of the 28 days before enrollment in the trial unlike other second-line trials. The primary endpoint of OS was significantly better in the regorafenib arm than in the placebo arm (10.6 vs. 7.8 months, respectively) (HR $=0.63$; 95\% CI: $0.50-0.79 ; p<0.0001$ ] [25]. PFS and TTP were also significantly better (Table 4). Regorafenib became the first drug to show efficacy as second-line therapy after progression on sorafenib compared with placebo. These results led to the additional approval of regorafenib as second-line therapy in 2017.

The success of the RESORCE trial can be attributed to the aggregate effects of the following 4 factors: (1) patients who discontinued sorafenib because of AEs were excluded from second- 
line therapy with regorafenib, leaving only patients with progressive disease (PD) on sorafenib; (2) imbalances between the active drug and placebo arms were avoided by including vascular invasion and EHS as separate stratification factors; (3) AFP, a strong prognostic factor, was also included as a stratification factor; and (4) only patients with adequate tolerance to sorafenib (patients able to take at least 400-mg sorafenib for at least 20 of the 28 days preceding the PD assessment) were selected. This trial design prevented dropouts related to adverse effects of regorafenib and minimized the effect of posttrial treatment after PD on regorafenib [25]. According to the results of the RESORCE trial, the median OS on regorafenib was 10.6 months (placebo: 7.8 months; $\mathrm{HR}=0.63 ; p<0.0001$ ). However, OS subanalysis showed that patients with a Child-Pugh score of 5 had significantly better results when starting sorafenib than patients with a score of 6 . This is because patients with a score of 5 could have been switched from TACE to sorafenib quickly if they were refractory to TACE and subsequently could have been switched to regorafenib if they were refractory to sorafenib; this early switch from ineffective TACE to systemic therapy will be an important strategy for improving survival under systemic therapy.

The median duration of treatment with sorafenib before enrollment into the RESORCE trial was a relatively long 7.8 months, indicating that most enrolled patients had slow PD that responded well to sorafenib. This led to questions regarding the efficacy of regorafenib in patients with rapid PD on sorafenib. However, a following report showed that the OS HR in RESORCE trial patients with rapid PD on sorafenib (TTP, 2.3 months) was 0.66 , indicating that regorafenib effectively prolonged OS even in patients with rapid PD on sorafenib [52].

\section{Sorafenib-Regorafenib Sequential Therapy}

Post hoc analysis of the RESORCE trial showed that sorafenib-regorafenib sequential therapy yielded good OS (26 months from the start of sorafenib treatment vs. 19.2 months for placebo) [52]. This is an extremely important finding because the long survival time of 26 months is similar to conventional TACE outcomes for intermediate-stage HCC [34]. The only phase III prospective trial reporting the OS for the TACE placebo arm is the BRISK-TA trial [34], which has the largest enrollment of any such trial in the world. Consequently, the outcomes of the placebo arm (26.1 months) in this trial could be considered the current global standard for TACE outcomes, with no selection bias whatsoever [34]. The patient population for this trial was as follows: 82\% early/intermediate stage (BCLC B: 59\%; BCLC A: 23\%; BCLC C: $17 \%$ ), with only $17 \%$ advanced-stage patients. By contrast, $86 \%$ of patients enrolled in the RESORCE trial had BCLC C advanced stage. Indirect comparison of these 2 cohorts showed that OS was comparable between TACE and sorafenib-regorafenib sequential therapy (26.1 vs. 26 months, respectively). Comparing the individual arms of 2 completely different randomized controlled trials (RCTs) (RESORCE and BRISK-TA trials) may not be appropriate; however, the OS value of 26.1 months is the placebo arm of a well-designed RCT, the BRISK-TA trial, and thus has no selection bias. The fact that OS was comparable between the 2 is particularly important because sorafenib-regorafenib sequential therapy is indicated for a population with more advanced disease (advanced-stage HCC). Although the patient population is highly selective, the finding that the median OS obtained with TACE (in intermediate-stage patients for whom it is indicated) can be obtained with sorafenib-regorafenib sequential therapy in patients with advanced-stage HCC is nonetheless very important [34, 52]. Similar or even better results regarding sorafenib-regorafenib sequential therapy in a clinical practice setting were recently published [53-57]. The results confirming the potential of sorafenibregorafenib sequential therapy to improve prognosis suggest that the appropriate time for starting sorafenib therapy needs to be re-evaluated. Conventional practice has been to switch from TACE to systemic therapy when the patient becomes refractory to TACE; however, it may become increasingly important to identify subgroups that tend to be refractory to TACE

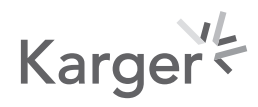


Kudo: Systemic Therapy for HCC

Table 5. Efficacy results of the phase 3 CELESTIAL trial (RECIST 1.1)

\begin{tabular}{lccl}
\hline & Cabozantinib $(n=470)$ & Placebo $(n=237)$ & HR, $p$ value \\
\hline OS, M (95\% CI) & $10.2(9.1-12.0)$ & $8.0(6.8-9.4)$ & $\begin{array}{l}\text { HR 0.76 (95\% CI: 0.63-0.92) } \\
p=0.0049\end{array}$ \\
PFS, M (95\% CI) & $5.2(4.0-5.5)$ & $1.9(1.9-1.9)$ & $\begin{array}{l}\text { HR } 0.44(95 \% \text { CI: 0.36-0.52) } \\
p<0.0001\end{array}$ \\
Objective response, $n(\%)^{*}$ & & & \\
CR & 0 & 0 & \\
PR & $18(4)$ & $1(<1)$ & \\
SD & $282(60)$ & $78(33)$ & \\
PD & $98(21)$ & $131(55)$ & \\
NE & $72(15)$ & $27(11)$ & \\
ORR, \% (95\% CI) & $4(2-6)$ & 33.4 & \\
DCR, \% & 64 & & \\
\end{tabular}

OS, overall survival; PFS, progression-free survival; TTP, time to progression; CR, complete response; PR, partial response; SD, stable disease; PD, progressive disease; NE, not evaluable. * Investigator assessment per RECIST 1.1.

and to start systemic therapy earlier [58-60] (i.e., while hepatic functional reserve is still Child-Pugh 5 before patients are found to be refractory to TACE).

Regarding safety and efficacy, there is no subgroup analysis by age in the RESORCE trial [25]. However, in the RESORCE trial, OS, TTP, and PFS HRs were similar in elderly patients ( $\geq 65$ years) and in nonelderly patients (OS HR: $0.74 ; 95 \%$ CI: $0.54-1.02$ vs. 0.65 ; $95 \%$ CI: 0.49-0.87; TTP HR: 0.51 ; $95 \%$ CI: $0.38-0.68$ vs. 0.46 ; $95 \%$ CI: $0.36-0.59$; and PFS HR: 0.50 ; $95 \%$ CI: $0.37-0.68$ vs. $0.45 ; 95 \%$ CI: $0.35-0.58$ ). There are no reports on the difference in safety and efficacy of regorafenib between elderly and nonelderly patients in real-world practice.

\section{Cabozantinib: Overview of the CELESTIAL Trial}

Cabozantinib is an oral multikinase inhibitor of VEGF, c-MET, RET, TIE2, FLT3, and the TAM family of receptor kinases (TYRO-3, AXL, and MER). When used as a second-line drug, it prolongs survival (compared with placebo) in patients with HCC who are refractory or intolerant to sorafenib $[28,61]$. A total of 707 patients with unresectable HCC were assigned to the cabozantinib or placebo arm in a 2:1 ratio. The median OS was significantly longer in the cabozantinib arm $(n=470 ; 10.2$ months; $95 \% \mathrm{CI}$ : 9.1-12.0) than in the placebo arm $(8.0$ months; 95\% CI: 6.8-9.4) (HR = 0.76; 95\% CI: 0.63-0.92; $p<0.001$ ) (Table 5) [28]. The median PFS was 5.2 months with cabozantinib and 1.9 months with placebo (HR $=0.44 ; 95 \% \mathrm{CI}$ : $0.36-0.52 ; p<0.001)$. The ORR per RECIST 1.1 was $4 \%$ in the cabozantinib arm and $<1 \%$ in the placebo arm ( $p=0.009)$ [28] (Table 5).

In the subgroup of patients whose previous systemic therapy was only sorafenib $(n=$ $486,69 \%$ ), the median OS was 11.3 months with cabozantinib and 7.2 months with placebo ( $\mathrm{HR}=0.70 ; 95 \% \mathrm{CI}: 0.55-0.88)$ and the median PFS was 5.5 months with cabozantinib and 1.9 months with placebo ( $\mathrm{HR}=0.40 ; 95 \% \mathrm{CI}: 0.32-0.50$ ). This trial also included patients who had received systemic therapy with one other drug besides sorafenib, thereby establishing cabozantinib as both a second- and third-line drug option.

Regarding safety, grade 3 or 4 AEs occurred in $68 \%$ of patients in the cabozantinib arm and in $36 \%$ in the placebo arm. The most common high-grade AEs were hand-foot skin syndrome ( $17 \%$ with cabozantinib vs. $0 \%$ with placebo), hypertension (16 vs. $2 \%$ ), increased 
Table 6. Efficacy results of the phase 3 REACH-2 trial (RECIST 1.1)

\begin{tabular}{llll}
\hline & Ramucirumab $(n=197)$ & Placebo $(n=95)$ & HR, $p$ value \\
\hline OS, M (95\% CI) & 8.5 & 7.3 & $\begin{array}{l}\text { HR } 0.710 \text { (95\% CI: 0.531-0.949) } \\
p=0.0199\end{array}$ \\
PFS, M (95\% CI) & 2.8 & 1.6 & $\begin{array}{l}\text { HR } 0.452 \text { (95\% CI: 0.339-0.603) } \\
p<0.001\end{array}$ \\
Objective response, $n(\%)^{*}$ & & & \\
CR & $0(0.0)$ & $0(0.0)$ & \\
PR & $9(4.6)$ & $1(1.1)$ & \\
SD & $109(55.3)$ & $36(37.9)$ & \\
PD & $66(33.5)$ & $18(50.5)$ & \\
NE & $13(6.6)$ & $1(10.5)$ & $p=0.1697$ \\
ORR (\%) & $9(4.6)$ & $37(38.9)$ & $p=0.0006$ \\
DCR (\%) & $118(59.9)$ & & \\
\hline
\end{tabular}

OS, overall survival; PFS, progression-free survival; TTP, time to progression; CR, complete response; PR, partial response; SD, stable disease; $\mathrm{PD}$, progressive disease; NE, not evaluable. ${ }^{*}$ Investigator assessment per RECIST 1.1.

AST level (12 vs. $7 \%$ ), fatigue (10 vs. $4 \%$ ), and diarrhea (10 vs. $2 \%)$. The phase III trial was not conducted in Japan; a bridging trial is currently underway, and approval in Japan is anticipated in 2021.

In the subgroup analysis of the CELESTIAL trial $[28,62]$, OS in elderly patients $(\geq 65$ years) was better in the cabozantinib arm than that in the placebo arm (11.1 vs. 8.3 months; $\mathrm{HR}=0.74 ; 95 \% \mathrm{CI}: 0.56-0.97$ ), and the results were similar in nonelderly patients ( $<65$ years) (9.6 vs. 7.7 months; HR $=0.8 ; 95 \%$ CI: $0.62-1.05$ ) [62]. Similarly, PFS in elderly patients ( $\geq 65$ years) was better in the cabozantinib arm than that in the placebo arm (5.4 vs. 2.0 months; $\mathrm{HR}=0.46$; 95\% CI: 0.35-0.59), which was consistent with the results in nonelderly patients (<65 years) (5.0 vs. 1.9 months; HR $=0.45$; 95\% CI: 0.35-0.57) [62].

\section{Ramucirumab: Overview of the REACH-2 Trial}

Ramucirumab is a recombinant human immunoglobulin IgG1 monoclonal antibody that inhibits VEGFR-2. It is an intravenous formulation that exerts its antitumor effects by inhibiting the activation of VEGFR-2 by preventing binding to VEGF-A, VEGF-C, and VEGF-D; this interferes with endothelial proliferation, migration, and survival and inhibits tumor angiogenesis.

The first phase III trial, the REACH trial, attempted to demonstrate that ramucirumab prolongs survival compared with placebo in patients with unresectable advanced HCC who are refractory or intolerant to sorafenib; the results were negative in the overall patient population [22]. However, subanalysis revealed that survival improved in the group with AFP levels $\geq 400 \mathrm{ng} / \mathrm{mL}$, which is a strong poor prognostic factor in HCC [63]. Therefore, a new phase III trial was conducted and termed the REACH-2 trial. The trial design did not differ much from the original REACH trial except for restricting enrollment to patients with AFP levels $\geq 400 \mathrm{ng} / \mathrm{mL}$ and including gross vascular invasion as a stratification factor. The results were positive, showing that ramucirumab prolonged the primary endpoint of OS $[29,64]$. Median OS in the ramucirumab arm was significantly longer than that in the placebo arm (8.5 vs. 7.3 months, respectively; $H R=0.710 ; p=0.0199$ ) (Table 6). In light of these results, the indication for ramucirumab was restricted to patients with sorafenib-refractory or intolerant HCC with AFP levels $\geq 400 \mathrm{ng} / \mathrm{mL}$.

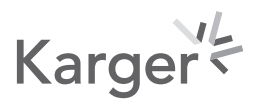


Table 7. RDI of molecular targeted agents

\begin{tabular}{|c|c|c|c|c|c|c|c|}
\hline \multirow{2}{*}{\multicolumn{2}{|c|}{$\frac{\text { Monoclonal antibody }}{\text { ramucirumab (median) }[29,66]}$}} & \multicolumn{6}{|l|}{ TKI } \\
\hline & & \multicolumn{2}{|c|}{ lenvatinib (mean) $[16,67]$} & \multicolumn{2}{|c|}{ sorafenib (mean) $[16,67]$} & \multicolumn{2}{|c|}{ regorafenib (mean) $[25,65]$} \\
\hline $\begin{array}{l}\text { Japanese } \\
\text { cohort }\end{array}$ & $\begin{array}{l}\text { Overall global } \\
\text { cohort }\end{array}$ & $\begin{array}{l}\text { Japanese } \\
\text { cohort }\end{array}$ & $\begin{array}{l}\text { Overall global } \\
\text { cohort }\end{array}$ & $\begin{array}{l}\text { Japanese } \\
\text { cohort }\end{array}$ & $\begin{array}{l}\text { Overall global } \\
\text { cohort }\end{array}$ & $\begin{array}{l}\text { Japanese } \\
\text { cohort }\end{array}$ & $\begin{array}{l}\text { Overall global } \\
\text { cohort }\end{array}$ \\
\hline $98 \%$ & $98 \%$ & $75 \%$ & $88 \%$ & $70 \%$ & $83 \%$ & $84 \%$ & $91 \%$ \\
\hline
\end{tabular}

RDI, relative dose intensity; TKI, tyrosine kinase inhibitor. RDI: ramucirumab (overall global cohort) = ramucirumab (Japanese cohort). RDI: ramucirumab (overall global/Japanese) > TKI (overall global cohort) > TKI (Japanese cohort).

Table 8. Ramucirumab: relative dose intensity, OS, and PFS among 3 age subgroups in pooled REACH-2/REACH (AFP $\geq 400$ ng/ $\mathrm{mL}$ )

\begin{tabular}{|c|c|c|c|c|c|c|}
\hline & \multicolumn{2}{|l|}{ Age $<65$ years } & \multicolumn{2}{|l|}{ Age $\geq 65$ to $<75$ years } & \multicolumn{2}{|l|}{ Age $\geq 75$ years } \\
\hline & $\begin{array}{l}\text { ram } \\
(n=171)\end{array}$ & placebo & $\begin{array}{l}\text { ram } \\
(n=93)\end{array}$ & $\begin{array}{l}\text { placebo } \\
(n=67)\end{array}$ & $\begin{array}{l}\text { ram } \\
(n=52)\end{array}$ & $\begin{array}{l}\text { placebo } \\
(n=28)\end{array}$ \\
\hline $\begin{array}{l}\text { Dose intensity median, \% } \\
\text { OS }\end{array}$ & 98.5 & 99.8 & 98.0 & 99.9 & 97.8 & 98.6 \\
\hline $\begin{array}{l}\text { Median (M) } \\
\operatorname{HR}(95 \% \mathrm{CI})\end{array}$ & $\begin{array}{c}8.2 \\
0.753(0.581-0.975)\end{array}$ & 4.8 & $\begin{array}{l}7.6 \\
0.602(0.419-0.866)\end{array}$ & 5.2 & $\begin{array}{l}8.9 \\
0.709(0.420-1.199)\end{array}$ & 6.3 \\
\hline PFS & & & & & & \\
\hline $\begin{array}{l}\text { Median (M) } \\
\text { HR }(95 \% \text { CI) }\end{array}$ & $\begin{array}{l}2.7 \\
0.613(0.472-0.796)\end{array}$ & 1.5 & $\begin{array}{l}2.8 \\
0.563(0.396-0.802)\end{array}$ & 1.8 & $\begin{array}{l}4.2 \\
0.480(0.282-0.817)\end{array}$ & 1.6 \\
\hline TTP & & & & & & \\
\hline $\begin{array}{l}\text { Median (M) } \\
\text { HR }(95 \% \text { CI) }\end{array}$ & $\begin{array}{l}2.8 \\
0.591(0.447-0.782)\end{array}$ & 1.5 & $\begin{array}{l}2.8 \\
0.555(0.377-0.818)\end{array}$ & 1.9 & $\begin{array}{l}4.2 \\
0.443(0.255-0.769)\end{array}$ & 2.0 \\
\hline
\end{tabular}

OS, overall survival; PFS, progression-free survival; TTP, time to progression; AFP, alpha-fetoprotein. This table is cited from Ref. [69].

Ramucirumab was better tolerated than TKIs, with an acceptable dose intensity of $98 \%$ (Table 7) [16, 25, 29, 65-67]. The relative dose intensity of any TKI is low in Japanese patients; however, the relative dose intensity of ramucirumab was similar between Japanese patients and an overall global cohort despite the fact that Japanese patients are more elderly (Table 7). Therefore, the dose intensity of ramucirumab is higher than that of any existing TKI (sorafenib, lenvatinib, regorafenib, or cabozantinib) in both the overall population and in the Japanese population [16, 25, 29, 65-68] (Table 7).

Regarding the efficacy of ramucirumab in pooled patients of the REACH (baseline AFP $\geq 400 \mathrm{ng} / \mathrm{mL}$ ) and REACH-2 trials, ramucirumab prolonged OS over placebo in patients $<65$ years (8.2 vs. 4.8 months; $\mathrm{HR}=0.753 ; 95 \% \mathrm{CI}$ : $0.581-0.975]$ ), in patients $\geq 65$ to $<75$ years (7.6 vs. 5.2 months; $\mathrm{HR}=0.602 ; 95 \%$ CI: $0.419-0.866$ ), and in patients $\geq 75$ years ( 8.9 vs. 6.6 months; $\mathrm{HR}=0.709 ; 95 \% \mathrm{CI}: 0.420-1.199$ ) [69]. In the 3 age subgroups, compared with placebo, ramucirumab also improved PFS ( $<65$ years: 2.7 vs. 1.5 months; $\mathrm{HR}=0.613$; $95 \% \mathrm{CI}$ : $0.472-0.796 ; \geq 65$ to $<75$ years, 2.8 vs. 1.8 months; $\mathrm{HR}=0.563 ; 95 \% \mathrm{CI}: 0.396-0.802$; and $>75$ years: 4.2 vs. 1.6 months; $\mathrm{HR}=0.480 ; 95 \% \mathrm{CI}: 0.282-0.817$ ) and TTP ( $<65$ years: 2.8 vs. 1.5 months; $\mathrm{HR}=0.591 ; 95 \% \mathrm{CI}: 0.447-0.782 ; \geq 65$ to $<75$ years: 2.8 vs. 1.9 months; $\mathrm{HR}=0.555$; 95\% CI: $0.377-0.818$; and $\geq 75$ years: 4.2 vs. 2.0 months; $\mathrm{HR}=0.443$; $95 \% \mathrm{CI}$ : $0.255-0.769$ ) [69] (Table 8). 
Kudo: Systemic Therapy for HCC

Regarding safety, the incidence of grade 3 or more treatment emergent AEs in the $\geq 75$-year subgroup was comparable with ramucirumab in the 2 younger subgroups $(<65$ and $\geq 65$ to $<75$ years) ( 54 and $60 \%$, respectively). AEs of special interest in the ramucirumab arm were comparable among the 3 age subgroups. A trend for a delay in the deterioration of symptoms as measured by patient-reported outcomes (FHSI-8) was similar among the 3 age subgroups [69].

\section{Immune Checkpoint Inhibitors}

\section{Nivolumab}

Nivolumab is the world's first recombinant human IgG4 monoclonal antibody specific for human PD-1. In a phase I/II trial in patients with advanced HCC (CheckMate 040 trial), the response rate for nivolumab was $20 \%$ (including 3 complete responses [CRs]) and the DCR was $64 \%$, which are extremely promising results. A unique feature of nivolumab is that its effects persist in responders [70]. These findings led to an expansion in trial enrollment. The updated results, which were presented at ASCO 2017, were promising, with an OS of 28.6 months for first-line therapy and 15 months for second-line therapy. Based on these results, nivolumab was approved by the FDA as a second-line agent for HCC after sorafenib.

The phase III CheckMate 459 trial, which compared nivolumab with sorafenib, showed that nivolumab did not improve OS over that achieved with sorafenib [17]. This failure might be attributable to posttrial treatment of the sorafenib arm with checkpoint inhibitors (20\%) such as nivolumab or pembrolizumab, which is also approved by the FDA as a second-line agent after sorafenib.

The CheckMate 459 trial did not include specific subgroup analysis of the safety and efficacy of nivolumab in elderly patients. However, OS was better in the nivolumab arm than in the sorafenib arm both in elderly patients ( $\geq 65$ years) and in nonelderly patients $(<65$ years) (HR $=0.88 ; 95 \%$ CI: 0.68-1.12 vs. $\mathrm{HR}=0.80 ; 95 \%$ CI: 0.63-1.02, respectively) [70].

\section{Pembrolizumab}

Pembrolizumab is a recombinant human IgG4 monoclonal antibody specific for human PD-1, similar to nivolumab. In a phase II trial in patients with HCC, it showed a response rate comparable to that of nivolumab (17\%) [71]. In addition, it was approved by the FDA as a second-line agent after sorafenib regardless of the reason for sorafenib discontinuation. The phase III KEYNOTE-240 trial comparing pembrolizumab with placebo failed to show that pembrolizumab improved OS over placebo [30]. The coprimary endpoints of this trial were OS and PFS, and there were 2 interim analyses resulting in the consumption of $\alpha$ value. Therefore, although OS in the pembrolizumab arm was 13.9 months (compared with 10.6 months in the placebo arm) (HR $=0.781 ; 95 \% \mathrm{CI}: 0.611-0.998 ; p=0.0238$ ), it did not reach the prespecified $p$ value of 0.0174 . In that sense, this trial was determined to be clinically positive but statistically negative.

The KEYNOTE-240 study did not perform a specific subgroup analysis of the safety and efficacy of pembrolizumab in elderly patients. However, OS was better in the pembrolizumab arm than in the placebo arm both in elderly patients ( $\geq 65$ years) and in nonelderly patients ( $<65$ years) (HR $=0.92 ; 95 \%$ CI: $0.65-1.30$ vs. HR $=0.75 ; 95 \%$ CI: 0.52-1.09). Similarly, PFS was better in the pembrolizumab arm than in the placebo arm both in elderly patients $(\geq 65$ years) and in nonelderly patients ( $<65$ years) ( $\mathrm{HR}=0.67 ; 95 \% \mathrm{CI}: 0.49-0.92$ vs. $\mathrm{HR}=0.89$; 95\% CI: 0.63-1.27).

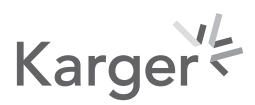


Table 9. Efficacy results of the phase 3 IMbrave 150 trial (RECIST 1.1)

\begin{tabular}{llll}
\hline & $\begin{array}{l}\text { Atezolizumab + bevacizumab } \\
(n=336)\end{array}$ & $\begin{array}{l}\text { Sorafenib } \\
(n=165)\end{array}$ & HR, $p$ value \\
\hline OS, M (95\% CI) & NE & $13.2(10.4-\mathrm{NE})$ & $\begin{array}{l}\text { HR } 0.58(95 \% \text { CI: } 0.42-0.79) \\
0.0006\end{array}$ \\
PFS, M (95\% CI) & $6.8(5.7-8.3)$ & $4.3(4.0-5.6)$ & $\begin{array}{l}\text { HR } 0.59(95 \% \text { CI: } 0.47-0.76) \\
<0.0001\end{array}$ \\
Objective response, $n(\%)^{*}$ & $(n=326)$ & $(n=159)$ & \\
CR & $18(6)$ & 0 & \\
PR & $71(22)$ & $19(12)$ & \\
SD & $151(46)$ & $69(43)$ & \\
PD & $64(20)$ & $39(25)$ & \\
ORR & $89(27)$ & $19(12)$ & $p<0.0001$ \\
DCR & $240(74)$ & $88(55)$ & $p<0.0001$ \\
\hline
\end{tabular}

NE, not estimable; OS, overall survival; PFS, progression-free survival; HR, hazard ratio; CR, complete response; PR, partial response; SD, stable disease; PD, progressive disease; DCR, disease control rate. ${ }^{*}$ Independent imaging review per RECIST 1.1 .

\section{Combination Immunotherapy with Atezolizumab plus Bevacizumab}

The phase III IMbrave150 study is an international multicenter phase III open-label study of patients with locally advanced or metastatic and/or unresectable HCC; this trial compared Atezo + Beva with sorafenib. Patients were allocated randomly (2:1 ratio) to the Atezo + Beva group (336 patients) or sorafenib group (165 patients). The coprimary endpoints were OS and PFS, as assessed by independent review facility (IRF) per RECIST v1.1. The stratification factors were geographic region (Asia excluding Japan vs. the rest of the world), ECOG PS 0 versus 1, macrovascular invasion and/or EHS (presence vs. absence), and serum AFP level ( $<400$ vs. $\geq 400 \mathrm{ng} / \mathrm{mL}$ ).

Success in meeting both the OS and PFS endpoints at the first interim analysis was surprising and heralded the arrival of a "new era of systemic therapy for HCC." Detailed results were described in the recently published literature [19].

The median OS was not estimable (NE) in the Atezo + Beva arm, whereas it was 13.2 months (95\% CI: 10.4-NE) in the sorafenib arm, with a HR of 0.58 (95\% CI: $0.42-0.79 ; p=$ 0.0006; Table 9). This combination strategy is the first (to date) to show a clinical benefit over sorafenib since 2007 . The IMbrave150 study reported a PFS (according to IRF per RECIST v1.1) of 6.8 months ( $95 \%$ CI: 5.7-8.3 months) for the Atezo + Beva arm and 4.3 months (95\% CI: 4.0-5.6 months) for the sorafenib arm, with a HR of 0.59 (95\% CI: $0.47-0.76 \% ; p<0.0001$ ).

The difference in ORR according to the IRF per RECIST v1.1 was significant (stratified $p$ value $<0.0001$ ): Atezo + Beva arm $=27 \%, 95 \% \mathrm{CI}$ : $23-33 \%$; sorafenib arm $=12 \%, 95 \% \mathrm{CI}$ : $7-18 \%)$. CR was achieved in 18 patients (6\%), which is quite promising. The median duration of response (DOR) of NE and the proportion (80\%) of responders with a DOR of $>6$ months by Atezo + Beva therapy indicate a considerable durable response to this treatment (Table 9).

Treatment-related AEs (grade 3 and 4) were more common in the sorafenib arm (46\%, 71/156) than in the Atezo + Beva arm (36\%, 117/329). Among AEs occurring at a rate of $\geq 10 \%$, those that commonly occurred in the sorafenib arm (diarrhea, hand-foot skin reaction, decreased appetite, hypertension, alopecia, and asthenia) were less common in the Atezo + Beva arm. Most surprisingly, immune-related AEs (except for infusion reactions in $10.9 \%$, autoimmune hemolytic anemia in $0.3 \%$, and adrenal insufficiency in $0.3 \%$ ) were rarely observed in the Atezo + Beva arm; this is not the case for ICIs such as nivolumab [17] or pembrolizumab [30]. In addition, Beva-related bleeding events in grade 3 or 4 were minimal, 


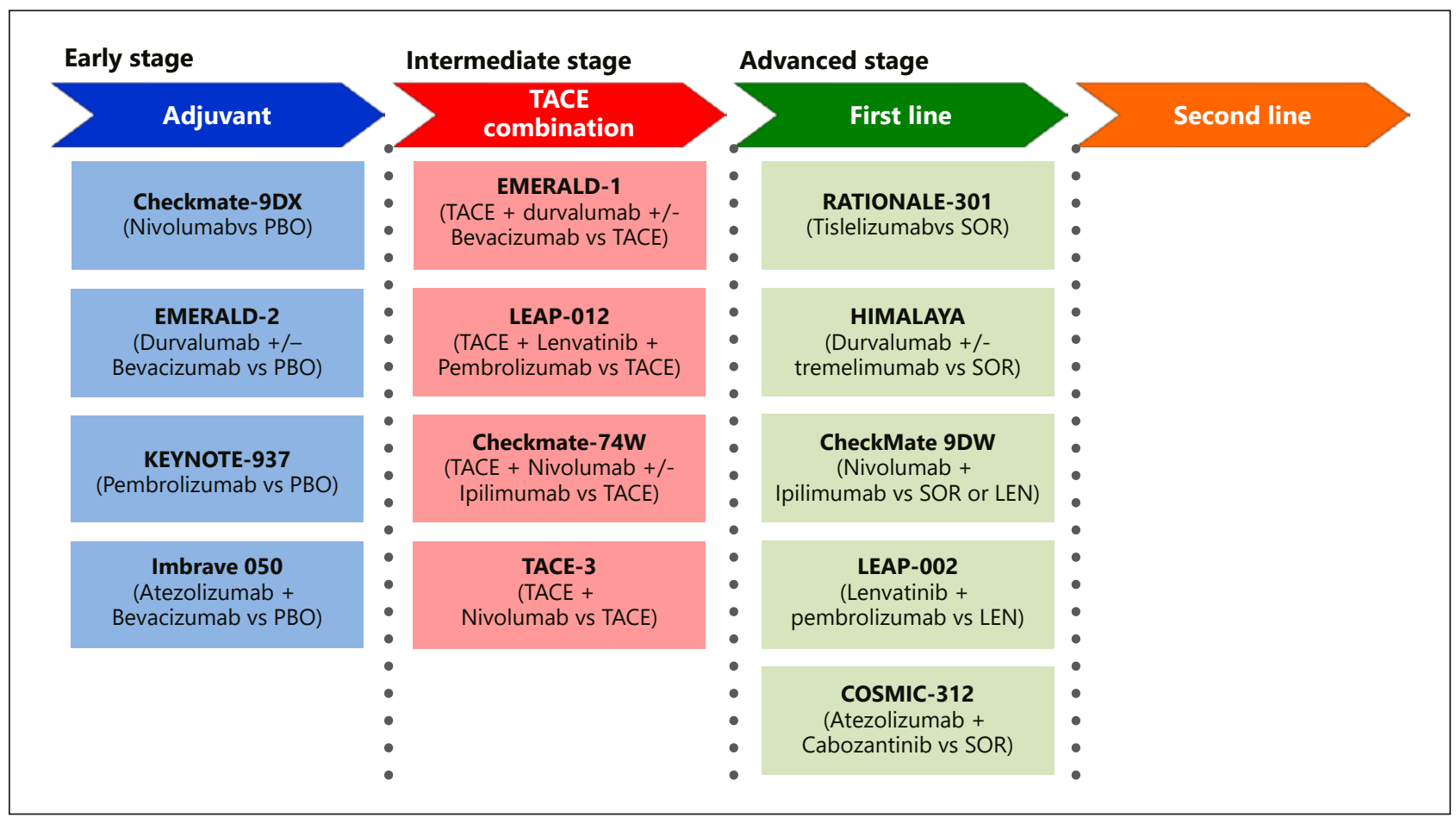

Fig. 2. Ongoing phase III trials in early, intermediate, and advanced stages. All of the trials are ICIs \pm antiVEGF/tyrosine kinase inhibitors. ICIs, immune checkpoint inhibitors.

occurring at a rate of $6.4 \%$. Taken together, the data suggest that the safety profile of Atezo + Beva is good.

The Atezo + Beva strategy may induce a paradigm change in the management of HCC, and after the successful phase III study, combination therapy with Atezo + Beva will be available in routine clinical practice worldwide as a first option, first-line therapy for HCC. In a subgroup analysis of the IMbrave150 trial, the median OS was better in the Atezo + Beva arm than in the sorafenib arm in elderly patients ( $\geq 65$ years) (NE vs. 14.9 months, HR $=0.58$; $95 \% \mathrm{CI}$ : $0.36-0.92$ ), and similar results were obtained in nonelderly patients ( $<65$ years) (NE vs. 11.4 months, HR $=0.59 ; 95 \%$ CI: 0.38-0.91) [72].

PFS was better in the Atezo + Beva arm than in the sorafenib arm in elderly patients ( $\geq 65$ years) (7.7 vs. 4.8 months, $\mathrm{HR}=0.63 ; 95 \% \mathrm{CI}$ : $0.45-0.89$ ), and similar results were obtained in nonelderly patients ( $<65$ years) (6.7 vs. 2.9 months, $\mathrm{HR}=0.50 ; 95 \% \mathrm{CI}: 0.36-0.71$ ). The ORR and CR rate in elderly patients and nonelderly patients were 26 versus $29 \%$ and 5 versus $6 \%$, respectively [72].

Atezo + Beva consistently delayed deterioration of patient-reported quality of life compared with sorafenib across the 2 age groups. The frequency and severity of observed AEs in the Atezo + Beva arm were consistent across the 2 age groups [72].

\section{Other Combination Immunotherapies}

\section{Lenvatinib plus Pembrolizumab}

Basic research studies show that lenvatinib and anti-PD-1 antibodies have synergistic effects $[73,74]$. Combination therapy with ICIs and MTAs will likely play a central role in the 
treatment of HCC going forward [75]. A phase Ib trial of lenvatinib plus pembrolizumab $(n=$ 100) showed favorable results [76]. This combination therapy yielded a median OS of 22.0 months (95\% CI: 20.4-NE), PFS of 9.3 months (95\% CI: 5.6-9.7), and ORR per mRECIST by independent imaging review of $46 \%$ (95\% CI: 36.0-56.3). A phase III trial (LEAP002) of this combination immunotherapy is currently ongoing (Table 1; Fig. 2).

\section{Durvalumab plus Tremelimumab}

The results of combination therapy with the PD-L1 antibody durvalumab (Durva) and the CTLA-4 antibody tremelimumab (Treme) were presented at ASCO 2020. Patients treated with Treme $300 \mathrm{mg} \times 1$ dose + Durva 1,500 mg Q4W arm $(n=65)$ showed a median OS of 18.73 months (95\% CI: 10.78-27.27), median PFS of 2.17 months (95\% CI: 1.91-5.42), and ORR per RECIST 1.1 of $24.0 \%$ (95\% CI: $14.9-35.3$ ). Therefore, this combination therapy is promising, and the phase III HIMALAYA trial of this combination (Treme $300 \mathrm{mg} \times 1$ dose + Durva 1,500 mg Q4W) is ongoing (Table 1; Fig. 2).

\section{Nivolumab plus Ipilimumab}

Combination therapy with the PD-1 antibody nivolumab (NIVO) and the CTLA-4 antibody ipilimumab (IPI) shows promising results in phase I/II CheckMate 040 trial [77]. Arm A, which received NIVO $1 \mathrm{mg} / \mathrm{kg}+$ IPI $3 \mathrm{mg} / \mathrm{kg}$ Q3W $\times 4$ followed by NIVO 240-mg flat dose Q2W $(n=50)$, showed a median OS of 22.8 months (95\% CI: 9.4-NE), and the ORR per RECIST v1.1 was $32 \%$ by blinded imaging review. Grade 3-4 TRAE of this combination occurred in $53 \%$ of patients. The most common immune-mediated AEs were rash, hepatitis, and adrenal insufficiency. Based on the results of phase I/II CheckMate 040 trial, this combination therapy was approved as second-line treatment after sorafenib by the FDA on March 11, 2020. The phase III CheckMate 9DW trial of this combination is currently ongoing (Table 1; Fig. 2).

Combination Immunotherapy in Early- and Intermediate-Stage HCC

Although many trials of immunotherapy in combination with TACE or in the adjuvant setting after resection or ablation failed [32-36, 78-81], trials of immunotherapy in combination with TACE or in the adjuvant setting after curative treatment are ongoing (Table 2; Fig. 2).

\section{Age Distribution for Phase III Trials}

Most of the positive phase III trials for unresectable HCC included elderly patients $>80$ years of age (Table 10). Patients $>65$ years account for approximately $50 \%$ of participants in recent trials (Table 10). In general, as described above, several multikinase inhibitors and antibodies specific for VEGF/VEGFR-2 and PD-1/PD-L1 are effective and show acceptable toxicity even in elderly patients if ECOG PS is maintained at 0-1 [82, 83].

\section{Possible Sequential Systemic Therapy}

The positive results of the IMbrave150 trial [19] led to the emergence of Atezo + Beva as the preferred option for first-line therapy. However, subsequent sequential MTA therapy is still important. In lung cancer, binding of the anti-PD-1 antibody nivolumab to CD8 ${ }^{+} \mathrm{T}$ cells persists for $>20$ months [84]. Therefore, even after PD following anti-PD-1/PD-L1 therapy, subsequent treatment with an MTA will change the tumor microenvironment (TME) from immune suppressive to immune permissive, resulting in a favorable TME similar to that 


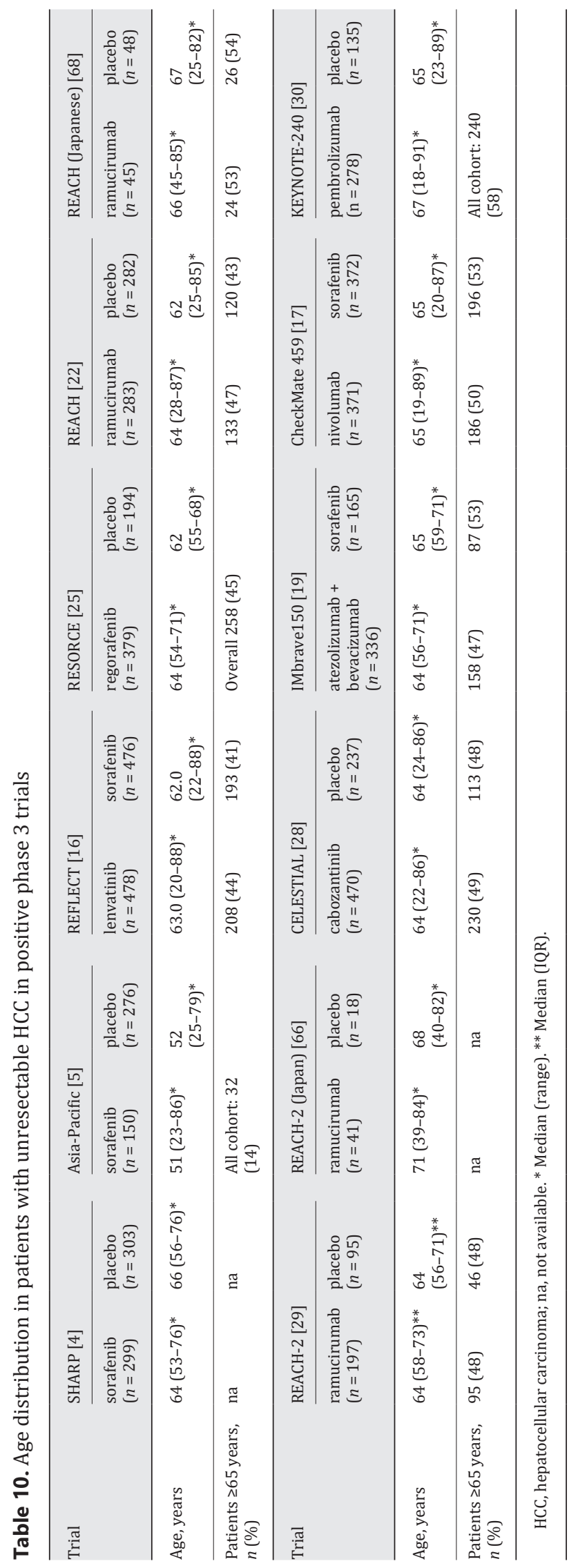




\begin{tabular}{|c|c|c|}
\hline \multirow[b]{2}{*}{ Liver Cancer } & \multicolumn{2}{|c|}{ Liver Cancer 2020;9:640-662 } \\
\hline & DOI: $10.1159 / 000511001$ & $\begin{array}{l}\text { (o) } 2020 \text { The Author(s). Published by S. Karger AG, Basel } \\
\text { www.karger.com/lic }\end{array}$ \\
\hline
\end{tabular}

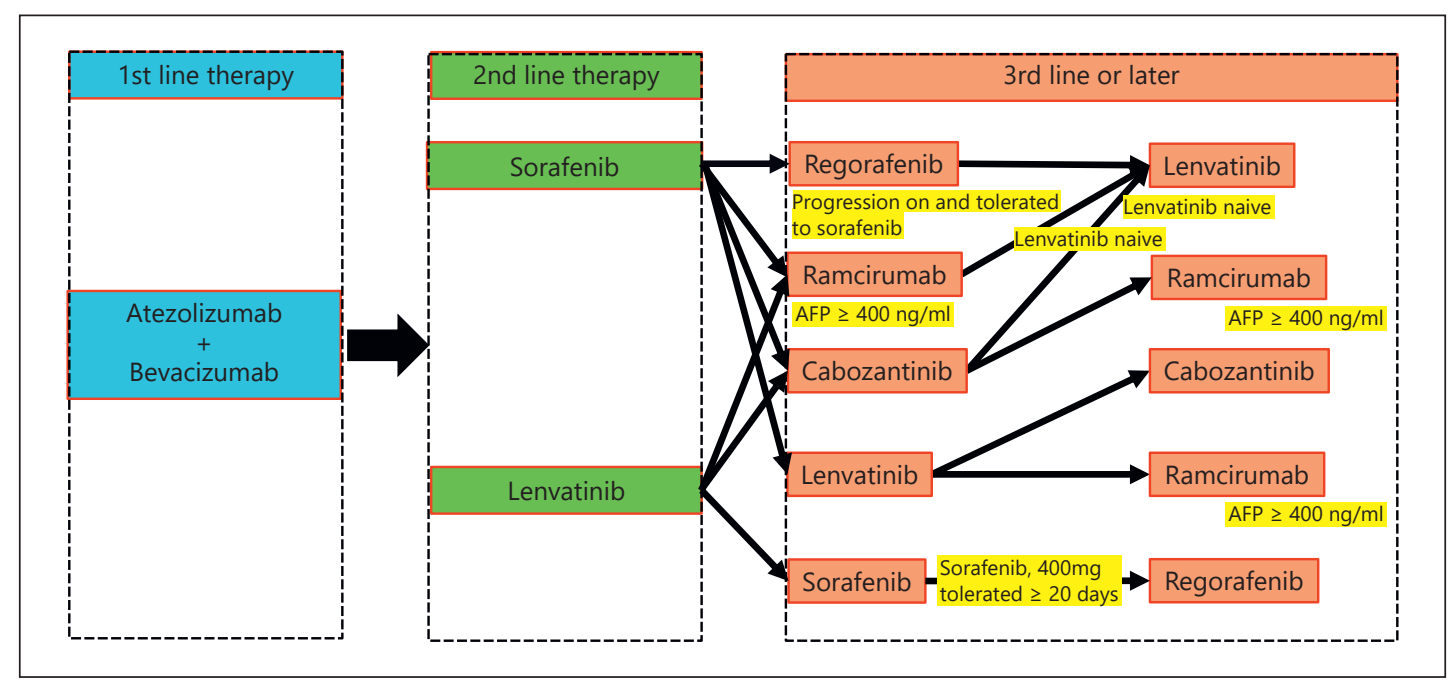

Fig. 3. Possible sequential systemic therapy for HCC after atezolizumab plus bevacizumab combination therapy. HCC, hepatocellular carcinoma; AFP, alpha-fetoprotein.

produced by combination immunotherapy with PD-1/PD-L1 antibody plus anti-VEGF/MTAs [85]. Sequential ICI-MTA therapy is thus expected to be effective [86].

In addition, ICIs are not effective in patients with WNT/ $\beta$-catenin-mutated HCC (immune exclusion subclass) [87-89]. The frequency of WNT/ $\beta$-catenin-mutated HCC is reported to be $20-30 \%[87,88,90]$. However, MTAs such as sorafenib are effective irrespective of WNT/ $\beta$ catenin mutation status [89].

Sequential MTAs should be effective after failure of ICI therapy and are thus important agents. There are many possible strategies for sequential therapy, as illustrated in Figure 3. To maximize the efficacy of MTAs, an early switch to systemic therapy from TACE is important.

\section{Systemic Therapy in Intermediate-Stage HCC}

The concept of TACE unsuitable or ineligible is currently widely accepted [59]. In the past, TACE failure/refractoriness [91] was used as an indicator for the timing of the switch from TACE to MTA. However, there are several conditions in which TACE is not only ineffective but also can be harmful. These conditions include the following: (1) conditions that easily become refractory to TACE, (2) conditions in which TACE causes deterioration of liver function to Child-Pugh class B, and (3) conditions that are unlikely to respond to TACE [59].

In this TACE unsuitable/ineligible patient population, lenvatinib is very effective [60, 92]. Particularly, lenvatinib-TACE sequential therapy can achieve complete response in many patients, including those with high tumor burden, such as up-to-seven criteria out tumor. This strategy is becoming a promising alternative to TACE not only in younger patients but also in elderly patients [58, 59]. 


\section{Systemic Therapy in Patients with Macrovascular Invasion}

All of the targeted therapy and immune checkpoint inhibitors are effective for vascular invasion in the pivotal studies $[4,5,16,17,19,25,28,29,70,71]$. However, hepatic arterial infusion chemotherapy can be an alternative treatment modality for major vascular invasion even in elderly patients or in patients with Child-Pugh B liver function $[13,93,94]$.

\section{Conclusions}

This article reviews recent advances in systemic therapy for HCC with special emphasis on the safety and efficacy of drugs in elderly patients. Lenvatinib, regorafenib, ramucirumab, and cabozantinib in addition to sorafenib are currently available as MTAs for the treatment of unresectable HCC. The increase in available MTAs for HCC will benefit many patients; however, drug selection and/or the sequence of the drugs will become more challenging. Positive results of combination immunotherapy with Atezo + Beva will result in a paradigm change in the treatment strategy of advanced HCC. These new drugs will likely benefit a wide range of patients with early-, intermediate-, and advanced-stage HCC, and many trials specific for these disease stages are ongoing (Tables 1, 2; Fig. 2).

Many patients aged $\geq 75$ years were enrolled in phase III trials. In this review, data from clinical trials as well as real-world data on efficacy, toxicity, tolerability, and quality of life in elderly patients indicate that these parameters are similar to those in younger patients, suggesting that available systemic therapies are beneficial for elderly patients if ECOG PS is maintained at 0 or 1 . Several real-world data, especially on sorafenib, support this conclusion; however, it should be cautious to generalize the subgroup analysis on safety and efficacy in elderly patients in real-world practice. In other words, these new agents should be carefully tested in elderly patients in real-world setting.

\section{Conflict of Interest Statement}

Lectures: Eisai, Bayer, MSD, Bristol-Myers Squibb, Lilly, and EA Pharma; Grants: Gilead Sciences, Taiho, Sumitomo Dainippon Pharma, Takeda, Otsuka, EA Pharma, AbbVie, and Eisai; advisory role: Eisai, Ono, MSD, Bristol-Myers Squibb, and Roche.

\section{Funding Sources}

The author did not receive any funding.

\section{Author Contributions}

M. Kudo conceived, wrote, and approved the final manuscript.

\section{References}

1 European Association for the Study of the Liver. Electronic address: easloffice@easloffice.eu; European Association for the Study of the Liver. EASL Clinical Practice Guidelines. Management of hepatocellular carcinoma. J Hepatol. 2018;69:182-236.

2 Marrero JA, Kulik LM, Sirlin CB, Zhu AX, Finn RS, Abecassis MM, et al. Diagnosis, staging, and management of hepatocellular carcinoma: 2018 practice guidance by the American Association for the Study of Liver Diseases. Hepatology. 2018;68(2):723-50. 


\begin{tabular}{|c|c|}
\hline Liver Cancer 2020;9:640-6 & \\
\hline DOI: 10.1159/000511001 & $\begin{array}{l}\text { (c) } 2020 \text { The Author(s). Published by S. Karger AG, Basel } \\
\text { www.karger.com/lic }\end{array}$ \\
\hline
\end{tabular}

Kudo: Systemic Therapy for HCC

3 Kokudo N, Takemura N, Hasegawa K, Takayama T, Kubo S, Shimada M, et al. Clinical practice guidelines for hepatocellular carcinoma: the Japan Society of Hepatology 2017 (4th JSH-HCC guidelines) a 2019 update. Hepatol Res. 2019;49(10):1109-13.

4 Llovet JM, Ricci S, Mazzaferro V, Hilgard P, Gane E, Blanc JF, et al. Sorafenib in advanced hepatocellular carcinoma. N Engl J Med. 2008;359(4):378-90.

5 Cheng AL, Kang YK, Chen Z, Tsao CJ, Qin S, Kim JS, et al. Efficacy and safety of sorafenib in patients in the AsiaPacific region with advanced hepatocellular carcinoma: a phase III randomised, double-blind, placebocontrolled trial. Lancet Oncol. 2009;10(1):25-34.

6 Kudo M. Targeted and immune therapies for hepatocellular carcinoma: predictions for 2019 and beyond. World J Gastroenterol. 2019;25(7):789-807.

7 Rimassa L, Pressiani T, Merle P. Systemic treatment options in hepatocellular carcinoma. Liver Cancer. 2019; 8(6):427-46.

8 Cheng AL, Kang YK, Lin DY, Park JW, Kudo M, Qin S, et al. Sunitinib versus sorafenib in advanced hepatocellular cancer: results of a randomized phase III trial. J Clin Oncol. 2013;31(32):4067-75.

9 Zhu AX, Rosmorduc O, Evans TR, Ross PJ, Santoro A, Carrilho FJ, et al. SEARCH: a phase III, randomized, doubleblind, placebo-controlled trial of sorafenib plus erlotinib in patients with advanced hepatocellular carcinoma. J Clin Oncol. 2015;33(6):559-66.

10 Johnson PJ, Qin S, Park JW, Poon RT, Raoul JL, Philip PA, et al. Brivanib versus sorafenib as first-line therapy in patients with unresectable, advanced hepatocellular carcinoma: results from the randomized phase III BRISK-FL study. J Clin Oncol. 2013;31(28):3517-24.

11 Cainap C, Qin S, Huang WT, Chung IJ, Pan H, Cheng Y, et al. Linifanib versus sorafenib in patients with advanced hepatocellular carcinoma: results of a randomized phase III trial. J Clin Oncol. 2015;33(2):172-9.

12 Abou-Alfa GK, Shi Q, Knox JJ, Kaubisch A, Niedzwiecki D, Posey J, et al. Assessment of treatment with sorafenib plus doxorubicin vs sorafenib alone in patients with advanced hepatocellular carcinoma: phase 3 CALGB 80802 randomized clinical trial. JAMA Oncol. 2019;5:1582-8.

13 Kudo M, Ueshima K, Yokosuka O, Ogasawara S, Obi S, Izumi N, et al. Sorafenib plus low-dose cisplatin and fluorouracil hepatic arterial infusion chemotherapy versus sorafenib alone in patients with advanced hepatocellular carcinoma (SILIUS): a randomised, open label, phase 3 trial. Lancet Gastroenterol Hepatol. 2018;3(6): 424-32.

14 Vilgrain V, Pereira H, Assenat E, Guiu B, Ilonca AD, Pageaux GP, et al. Efficacy and safety of selective internal radiotherapy with yttrium-90 resin microspheres compared with sorafenib in locally advanced and inoperable hepatocellular carcinoma (SARAH): an open-label randomised controlled phase 3 trial. Lancet Oncol. 2017;18:1624-36.

15 Chow PKH, Gandhi M, Tan S-B, Khin MW, Khasbazar A, Ong J, et al. SIRveNIB: selective internal radiation therapy versus sorafenib in Asia-Pacific patients with hepatocellular carcinoma. J Clin Oncol. 2018;36(19): 1913-21.

16 Kudo M, Finn RS, Qin S, Han K-H, Ikeda K, Piscaglia F, et al. Lenvatinib versus sorafenib in first-line treatment of patients with unresectable hepatocellular carcinoma: a randomised phase 3 non-inferiority trial. Lancet. 2018;391(10126):1163-73.

17 Yau T, Park JW, Finn RS, Cheng AL, Mathurin P, Edeline J, et al. CheckMate 459: a randomized, multi-center phase III study of nivolumab vs sorafenib as first-line treatment in patients with advanced hepatocellular carcinoma. Ann Oncol. 2019;30(Suppl 5):v874-5.

18 Ricke J, Klümpen HJ, Amthauer H, Bargellini I, Bartenstein P, de Toni EN, et al. Impact of combined selective internal radiation therapy and sorafenib on survival in advanced hepatocellular carcinoma. J Hepatol. 2019; 71(6):1164-74.

19 Finn RS, Qin S, Ikeda M, Galle PR, Ducreux M, Kim TY, et al. Atezolizumab plus bevacizumab in unresectable hepatocellular carcinoma. N Engl J Med. 2020;382(20):1894-905.

20 Llovet JM, Decaens T, Raoul JL, Boucher E, Kudo M, Chang C, et al. Brivanib in patients with advanced hepatocellular carcinoma who were intolerant to sorafenib or for whom sorafenib failed: results from the randomized phase III BRISK-PS study. J Clin Oncol. 2013;31(28):3509-16.

21 Zhu AX, Kudo M, Assenat E, Cattan S, Kang YK, Lim HY, et al. Effect of everolimus on survival in advanced hepatocellular carcinoma after failure of sorafenib: the EVOLVE-1 randomized clinical trial. JAMA. 2014;312(1): 57-67.

22 Zhu AX, Park JO, Ryoo BY, Yen CJ, Poon R, Pastorelli D, et al. Ramucirumab versus placebo as second-line treatment in patients with advanced hepatocellular carcinoma following first-line therapy with sorafenib (REACH): a randomised, double-blind, multicentre, phase 3 trial. Lancet Oncol. 2015;16(7):859-70.

23 Kudo M, Moriguchi M, Numata K, Hidaka H, Tanaka H, Ikeda M, et al. S-1 versus placebo in patients with sorafenib-refractory advanced hepatocellular carcinoma (S-CUBE): a randomised, double-blind, multicentre, phase 3 trial. Lancet Gastroenterol Hepatol. 2017;2(6):407-17.

24 Abou-Alfa GK, Qin S, Ryoo B-Y, Lu S-N, Yen C-J, Feng Y-H, et al. Phase III randomized study of second line ADI-PEG 20 plus best supportive care versus placebo plus best supportive care in patients with advanced hepatocellular carcinoma. Ann Oncol. 2018;29(6):1402-8.

25 Bruix J, Qin S, Merle P, Granito A, Huang YH, Bodoky G, et al. Regorafenib for patients with hepatocellular carcinoma who progressed on sorafenib treatment (RESORCE): a randomised, double-blind, placebocontrolled, phase 3 trial. Lancet. 2017;389(10064):56-66. 


\begin{tabular}{l}
\hline Liver Cancer 2020;9:640-662 \\
\hline DOI: 10.1159/000511001
\end{tabular}

Kudo: Systemic Therapy for HCC

26 Rimassa L, Assenat E, Peck-Radosavljevic M, Pracht M, Zagonel V, Mathurin P, et al. Tivantinib for second-line treatment of MET-high, advanced hepatocellular carcinoma (METIV-HCC): a final analysis of a phase 3, randomised, placebo-controlled study. Lancet Oncol. 2018;19(5):682-93.

27 Merle P, Blanc J-F, Phelip J-M, Pelletier G, Bronowicki J-P, Touchefeu Y, et al. Doxorubicin-loaded nanoparticles for patients with advanced hepatocellular carcinoma after sorafenib treatment failure (RELIVE): a phase 3 randomised controlled trial. Lancet Gastroenterol Hepatol. 2019;4(6):454-65.

28 Abou-Alfa GK, Meyer T, Cheng AL, El-Khoueiry AB, Rimassa L, Ryoo BY, et al. Cabozantinib in patients with advanced and progressing hepatocellular carcinoma. N Engl J Med. 2018;379(1):54-63.

29 Zhu AX, Kang YK, Yen CJ, Finn RS, Galle PR, Llovet JM, et al. Ramucirumab after sorafenib in patients with advanced hepatocellular carcinoma and increased alpha-fetoprotein concentrations (REACH-2): a randomised, double-blind, placebo-controlled, phase 3 trial. Lancet Oncol. 2019;20:282-96.

30 Finn RS, Ryoo B-Y, Merle P, Kudo M, Bouattour M, Lim HY, et al. Pembrolizumab as second-line therapy in patients with advanced hepatocellular carcinoma in KEYNOTE-240: a randomized, double-blind, phase III trial. J Clin Oncol. 2020;38(3):193-202.

31 Kudo M, Ueshima K, Ikeda M, Torimura T, Tanabe N, Aikata H, et al. Randomised, multicentre prospective trial of transarterial chemoembolisation (TACE) plus sorafenib as compared with TACE alone in patients with hepatocellular carcinoma: TACTICS trial. Gut. 2020;69(8):1492-501.

32 Kudo M, Imanaka K, Chida N, Nakachi K, Tak WY, Takayama T, et al. Phase III study of sorafenib after transarterial chemoembolisation in Japanese and Korean patients with unresectable hepatocellular carcinoma. Eur J Cancer. 2011;47(14):2117-27.

33 Lencioni R, Montal R, Torres F, Park JW, Decaens T, Raoul JL, et al. Objective response by mRECIST as a predictor and potential surrogate end-point of overall survival in advanced HCC. J Hepatol. 2017;66(6):1166-72.

34 Kudo M, Han G, Finn RS, Poon RT, Blanc JF, Yan L, et al. Brivanib as adjuvant therapy to transarterial chemoembolization in patients with hepatocellular carcinoma: a randomized phase III trial. Hepatology. 2014;60(5): 1697-707.

35 Kudo M, Cheng A-L, Park J-W, Park JH, Liang P-C, Hidaka H, et al. Orantinib versus placebo combined with transcatheter arterial chemoembolisation in patients with unresectable hepatocellular carcinoma (ORIENTAL): a randomised, double-blind, placebo-controlled, multicentre, phase 3 study. Lancet Gastroenterol Hepatol. 2018;3(1):37-46.

36 Meyer T, Fox R, Ma YT, Ross PJ, James MW, Sturgess R, et al. Sorafenib in combination with transarterial chemoembolisation in patients with unresectable hepatocellular carcinoma (TACE 2): a randomised placebocontrolled, double-blind, phase 3 trial. Lancet Gastroenterol Hepatol. 2017;2(8):565-75.

37 Cancer Information Services, National Cancer Center, Japan. http://gdb.ganjoho.jp/graph_db/ gdb1 showData $=\&$ dataType $=20 \&$ graphId $=105 \&$ totalTarget $=10 \&$ year $=2015 \&$ years $=2018 \&$ avgStep $=\&$ ageSybt $=0$ \&ageSt=009\&ageEd=A85\& currentAge $=0 \&$ smTypes $=8 \&$ sm Type $=8 \&$ sexType $=2 \&$ stage $=0$.

38 Cancer Information Services, National Cancer Center, Japan. http://gdb.ganjoho.jp/graph_db/ gdb1 showData $=\&$ dataType $=30 \&$ graphId $=105 \&$ totalTarget $=10 \&$ year $=2015 \&$ years $=2015 \&$ avgStep $=\&$ ageSybt=0\&ageSt=009\&ageEd=A85\&currentAge=0\&smTypes $=8 \&$ sm Type=8\&sexType $=2 \&$ stage $=0$.

39 Jo M, Yasui K, Kirishima T, Shima T, Niimi T, Katayama T, et al. Efficacy and safety of sorafenib in very elderly patients aged 80 years and older with advanced hepatocellular carcinoma. Hepatol Res. 2014;44(13):132938.

40 Ziogas DC, Papadatos-Pastos D, Thillai K, Korantzis I, Chowdhury R, Suddle A, et al. Efficacy and safety of sorafenib in patients with advanced hepatocellular carcinoma: age is not a problem. Eur J Gastroenterol Hepatol. 2017;29(1):48-55.

41 Nishikawa H, Takeda H, Tsuchiya K, Joko K, Ogawa C, Taniguchi H, et al. Sorafenib therapy for BCLC stage B/C hepatocellular carcinoma; clinical outcome and safety in aged patients: a multicenter study in Japan. J Cancer. 2014;5(7):499-509.

42 Williet N, Clavel L, Bourmaud A, Verot C, Bouarioua N, Roblin X, et al. Tolerance and outcomes of sorafenib in elderly patients treated for advanced hepatocellular carcinoma. Dig Liver Dis. 2017;49(9):1043-9.

43 Morimoto M, Numata K, Kondo M, Hidaka H, Takada J, Shibuya A, et al. Higher discontinuation and lower survival rates are likely in elderly Japanese patients with advanced hepatocellular carcinoma receiving sorafenib. Hepatol Res. 2011;41(4):296-302.

44 Montella L, Addeo R, Cennamo G, Vincenzi B, Palmieri R, Sperlongano P, et al. Sorafenib in elderly patients with advanced hepatocellular carcinoma: a case series. Oncology. 2013;84(5):265-72.

45 Ikeda K, Kudo M, Kawazoe S, Osaki Y, Ikeda M, Okusaka T, et al. Phase 2 study of lenvatinib in patients with advanced hepatocellular carcinoma. J Gastroenterol. 2017;52(4):512-9.

46 Kudo M. Extremely high objective response rate of lenvatinib: its clinical relevance and changing the treatment paradigm in hepatocellular carcinoma. Liver Cancer. 2018;7(3):215-24.

47 Kudo M. Objective response by mRECIST is an independent prognostic factor of overall survival in systemic therapy for hepatocellular carcinoma. Liver Cancer. 2019;8:73-7.

48 Kudo M. Lenvatinib may drastically change the treatment landscape of hepatocellular carcinoma. Liver Cancer. 2018;7:1-19.

49 Tada T, Kumada T, Hiraoka A, Michitaka K, Atsukawa M, Hirooka M, et al. Safety and efficacy of lenvatinib in elderly patients with unresectable hepatocellular carcinoma: a multicenter analysis with propensity score matching. Hepatol Res. 2020;50(1):75-83. 


\begin{tabular}{|c|c|}
\hline Liver Cancer 2020;9:640-6 & \\
\hline DOI: 10.1159/000511001 & $\begin{array}{l}\text { (c) } 2020 \text { The Author(s). Published by S. Karger AG, Basel } \\
\text { www.karger.com/lic }\end{array}$ \\
\hline
\end{tabular}

Kudo: Systemic Therapy for HCC

50 Tsuchiya K, Kurosaki M, Kaneko S, Wang W, Kirino S, Hayakawa Y, et al. Clinical outcome of tyrosine kinase inhibitors in the geriatric patients with unresectable hepatocellular carcinoma. Hepatology. 2019;70(Suppl 1):891.

51 Wilhelm SM, Dumas J, Adnane L, Lynch M, Carter CA, Schütz G, et al. Regorafenib (BAY 73-4506): a new oral multikinase inhibitor of angiogenic, stromal and oncogenic receptor tyrosine kinases with potent preclinical antitumor activity. Int J Cancer. 2011;129(1):245-55.

52 Finn RS, Merle P, Granito A, Huang YH, Bodoky G, Pracht M, et al. Outcomes of sequential treatment with sorafenib followed by regorafenib for HCC: additional analyses from the phase III RESORCE trial. J Hepatol. 2018;69(2):353-8.

53 Ogasawara S, Ooka Y, Itokawa N, Inoue M, Okabe S, Seki A, et al. Sequential therapy with sorafenib and regorafenib for advanced hepatocellular carcinoma: a multicenter retrospective study in Japan. Invest New Drugs. 2020;38(1):172-80.

54 Hiraoka A, Kumada T, Atsukawa M, Hirooka M, Tsuji K, Ishikawa T, et al. Important clinical factors in sequential therapy including lenvatinib against unresectable hepatocellular carcinoma. Oncology. 2019;97(5):277-85.

55 Yoo C, Park JW, Kim YJ, Kim DY, Yu SJ, Lim TS, et al. Multicenter retrospective analysis of the safety and efficacy of regorafenib after progression on sorafenib in Korean patients with hepatocellular carcinoma. Invest New Drugs. 2019;37(3):567-72.

56 Wang W, Tsuchiya K, Kurosaki M, Yasui Y, Inada K, Kirino S, et al. Sorafenib-regorafenib sequential therapy in Japanese patients with unresectable hepatocellular carcinoma-relative dose intensity and post-regorafenib therapies in real world practice. Cancers. 2019;11(10):1517.

57 Yoo C, Byeon S, Bang Y, Cheon J, Kim JW, Kim JH, et al. Regorafenib in previously treated advanced hepatocellular carcinoma: impact of prior immunotherapy and adverse events. Liver Int. 2020;40(9):2263-71.

58 Kudo M. A new era of systemic therapy for intermediate and advanced stage hepatocellular carcinoma. Hepatobil Surg Nutr. 2020;9(4):530-3.

59 Kudo M. A new treatment option for intermediate-stage hepatocellular carcinoma with high tumor burden: initial lenvatinib therapy with subsequent selective TACE. Liver Cancer. 2019;8(5):299-311.

60 Kudo M, Ueshima K, Chan S, Minami T, Chishina H, Aoki T, et al. Lenvatinib as an initial treatment in patients with intermediate-stage hepatocellular carcinoma beyond up-to-seven criteria and Child-Pugh A liver function: a proof-of-concept study. Cancers. 2019;11(8):1084.

61 Kudo M. Cabozantinib as a second-line agent in advanced hepatocellular carcinoma. Liver Cancer. 2018;7(2): 123-33.

62 Rimassa L, Blanc JF, Klumpen HJ, Zagonel V, Tran A, Kim SC, et al. Outcomes based on age in the phase 3 CELESTIAL trial of cabozantinib versus placebo in patients with advanced hepatocellular carcinoma. ASCO annual meeting. June 1-5, 2018.

63 Galle PR, Foerster F, Kudo M, Chan SL, Llovet JM, Qin S, et al. Issue cover. Liver Int. 2019;39(12):2214-29.

64 Kudo M. Ramucirumab as second-line systemic therapy in hepatocellular carcinoma. Liver Cancer. 2018; 7(4): 305-11.

65 Yokosuka O, Meinhardt G, Bruix J. Regorafenib as second-line treatment for patients with HCC who progressed on sorafenib: Japanese subgroup analysis of the phase 3 RESORCE trial. JSH annual meeting. 2017.

66 Kudo M, Okusaka T, Motomura K, Ohno I, Morimoto M, Seo S, et al. Ramucirumab after prior sorafenib in patients with advanced hepatocellular carcinoma and elevated alpha-fetoprotein: Japanese subgroup analysis of the REACH-2 trial. J Gastroenterol. 2020;55(6):627-39.

67 Yamashita T, Kudo M, Ikeda K, Izumi N, Tateishi R, Ikeda M, et al. REFLECT-a phase 3 trial comparing efficacy and safety of lenvatinib to sorafenib for the treatment of unresectable hepatocellular carcinoma: an analysis of Japanese subset. J Gastroenterol. 2020;55(1):113-22.

68 Kudo M, Hatano E, Ohkawa S, Fujii H, Masumoto A, Furuse J, et al. Ramucirumab as second-line treatment in patients with advanced hepatocellular carcinoma: Japanese subgroup analysis of the REACH trial. J Gastroenterol. 2017;52(4):494-503.

69 Kudo M, Galle PR, Llovet JM, Finn RS, Vogel A, Motomura K, et al. Ramucirumab in elderly patients with hepatocellular carcinoma and elevated alpha-fetoprotein after sorafenib in REACH and REACH-2. Liver Int. 2020. 10.1111/liv.14462.

70 El-Khoueiry AB, Sangro B, Yau T, Crocenzi TS, Kudo M, Hsu C, et al. Nivolumab in patients with advanced hepatocellular carcinoma (CheckMate 040): an open-label, non-comparative, phase 1/2 dose escalation and expansion trial. Lancet. 2017;389(10088):2492-502.

71 Zhu AX, Finn RS, Edeline J, Cattan S, Ogasawara S, Palmer D, et al. Pembrolizumab in patients with advanced hepatocellular carcinoma previously treated with sorafenib (KEYNOTE-224): a non-randomised, open-label phase 2 trial. Lancet Oncol. 2018;19(7):940-52.

72 Li D, Toh HC, Merle P, Tsuchiya K, Hernandez S, Shao H, et al. Atezolizumab + bevacizumab vs sorafenib for unresectable hepatocellular carcinoma (HCC): results from older adults enrolled in IMbrave150. ESMO-GI. July 1-4, 2020.

73 Kudo M. Immune checkpoint inhibition in hepatocellular carcinoma: basics and ongoing clinical trials. Oncology. 2017;92(Suppl 1):50-62.

74 Kato Y, Tabata K, Kimura T, Yachie-Kinoshita A, Ozawa Y, Yamada K, et al. Lenvatinib plus anti-PD-1 antibody combination treatment activates CD8+ T cells through reduction of tumor-associated macrophage and activation of the interferon pathway. PLoS One. 2019;14(2):e0212513. 


\begin{tabular}{|c|c|}
\hline Liver Cancer 2020;9:640-6 & \\
\hline DOI: 10.1159/000511001 & $\begin{array}{l}\text { (c) } 2020 \text { The Author(s). Published by S. Karger AG, Basel } \\
\text { www.karger.com/lic }\end{array}$ \\
\hline
\end{tabular}

Kudo: Systemic Therapy for HCC

75 Kudo M. Combination cancer immunotherapy in hepatocellular carcinoma. Liver Cancer. 2018;7:20-7.

76 Finn RS, Ikeda M, Zhu AX, Sung MW, Baron AD, Kudo M, et al. Phase Ib study of lenvatinib plus pembrolizumab in patients with unresectable hepatocellular carcinoma. J Clin Oncol. 2020;38(26):2960-70.

77 Yau T, Kang YK, Kim TY, El-Khoueiry AB, Santoro A, Sangro B, et al. Nivolumab plus ipilimumab in advanced hepatocellular carcinoma previously treated with sorafenib (CheckMate 040): a randomized clinical trial. JAMA Oncol. 2020.

78 Yoshida H, Shiratori Y, Kudo M, Shiina S, Mizuta T, Kojiro M, et al. Effect of vitamin K2 on the recurrence of hepatocellular carcinoma. Hepatology. 2011;54(2):532-40.

79 Okita K, Izumi N, Matsui O, Tanaka K, Kaneko S, Moriwaki H, et al. Peretinoin after curative therapy of hepatitis C-related hepatocellular carcinoma: a randomized double-blind placebo-controlled study. J Gastroenterol. 2015;50(2):191-202.

80 Bruix J, Takayama T, Mazzaferro V, Chau GY, Yang J, Kudo M, et al. Adjuvant sorafenib for hepatocellular carcinoma after resection or ablation (STORM): a phase 3, randomised, double-blind, placebo-controlled trial. Lancet Oncol. 2015;16(13):1344-54.

81 Tak WY, Lin S-M, Wang Y, Zheng J, Vecchione A, Park SY, et al. Phase III HEAT study adding lyso-thermosensitive liposomal doxorubicin to radiofrequency ablation in patients with unresectable hepatocellular carcinoma lesions. Clin Cancer Res. 2018;24(1):73-83.

82 Cho E, Cho HA, Jun CH, Kim HJ, Cho SB, Choi SK. A review of hepatocellular carcinoma in elderly patients focused on management and outcomes. In Vivo. 2019;33(5):1411-20.

83 Kapacee ZA, McNamara MG, de Liguori Carino N, Lamarca A, Valle JW, Hubner RA. Systemic therapies in advanced hepatocellular carcinoma: how do older patients fare? Eur J Surg Oncol. 2020; S07487983(20)30369-3.

84 Osa A, Uenami T, Koyama S, Fujimoto K, Okuzaki D, Takimoto T, et al. Clinical implications of monitoring nivolumab immunokinetics in non-small cell lung cancer patients. JCI Insight. 2018;3(19):e59125.

85 Kudo M. Scientific rationale for combined immunotherapy with PD-1/PD-L1 antibodies and VEGF inhibitors in advanced hepatocellular carcinoma. Cancers. 2020;12(5):1089.

86 Joerger M, Güller U, Bastian S, Driessen C, von Moos R. Prolonged tumor response associated with sequential immune checkpoint inhibitor combination treatment and regorafenib in a patient with advanced pretreated hepatocellular carcinoma. J Gastrointest Oncol. 2019;10(2):373-8.

87 Llovet JM, Montal R, Sia D, Finn RS. Molecular therapies and precision medicine for hepatocellular carcinoma. Nat Rev Clin Oncol. 2018;15(10):599-616.

88 Pinyol R, Sia D, Llovet JM. Immune exclusion-Wnt/CTNNB1 class predicts resistance to immunotherapies in HCC. Clin Cancer Res. 2019;25(7):2021-3.

89 Harding JJ, Nandakumar S, Armenia J, Khalil DN, Albano M, Ly M, et al. Prospective genotyping of hepatocellular carcinoma: clinical implications of next-generation sequencing for matching patients to targeted and immune therapies. Clin Cancer Res. 2019;25(7):2116-26.

90 Torbenson MS, Ng IOL, Park YN, Roncalli M, Sakamoto M. Hepatocellular carcinoma. In: WHO Classification of Tumours, 5th Edition, "Digestive System Tumours". Geneva, Switzerland: WHO Press; 2019. p. pp229-239.

91 Kudo M, Matsui O, Izumi N, Kadoya M, Okusaka T, Miyayama S, et al. Transarterial chemoembolization failure/ refractoriness: JSH-LCSGJ criteria 2014 update. Oncology. 2014;87(Suppl 1):22-31.

92 Kudo M, Ueshima K, Aikata H, et al. Association between tumor response by mRECIST and overall survival in patients with poorly differentiated HCC in REFLECT study.

93 Ueshima K, Ogasawara S, Ikeda M, Yasui Y, Terashima T, Yamashita T, et al. Hepatic arterial infusion chemotherapy versus sorafenib in patients with advanced hepatocellular carcinoma. Liver Cancer. 2020;9:583-95.

94 He M, Li Q, Zou R, Shen J, Fang W, Tan G, et al. Sorafenib plus hepatic arterial infusion of oxaliplatin, fluorouracil, and leucovorin vs sorafenib alone for hepatocellular carcinoma with portal vein invasion: a randomized clinical trial. JAMA Oncol. 2019;5(7):953-60. 